\title{
Scuttle flies (Diptera: Phoridae) of Vitosha Mt (Bulgaria)
}

\author{
Mario Langourov \\ National Museum of Natural History, Bulgarian Academy of Sciences, 1 Tsar Osvoboditel Blvd, 1000 Sofia, Bulgaria, \\ langourov@nmnhs.com $\nabla$; https://orcid.org/0000-0001-6756-3420〔ك
}

\begin{abstract}
Two hundred and one species of the family have been established on the territory of the Vitosha Mt. The genera Gymnoptera, Peromitra, Plectanocnema and Tubicera have been recorded for the first time on the Balkan Peninsula and Menozziola - in Bulgaria. Sixty-nine species are new for the Balkan Peninsula and additional thirty-two - for Bulgaria. On the basis of the established number of species, it can be concluded that Vitosha Mountain is an area with a significant scuttle fly diversity. Some new data are established about flower visiting of some scuttle fly species.
\end{abstract}

Keywords: Balkan Peninsula, Bulgaria, flower visiting, new data, Phoridae, Vitosha Mt

\section{Introduction}

Vitosha Mt is located in southwestern Bulgaria, south of Sofia - between $42^{\circ} 28^{\prime}$ and $42^{\circ} 40^{\prime} \mathrm{N}$ and $23^{\circ} 10^{\prime}$ and $23^{\circ} 22^{\prime} \mathrm{E}$, and belongs to the Kraishte-Srednogorie mountain system. It is surrounded by the Pernik Kettle (750 $\mathrm{m}$ above sea level), Sofia Kettle $(550 \mathrm{~m})$ and Samokov Kettle $(1000 \mathrm{~m})$ and the low mountains Lyulin, Verila and Plana, with which it has an orographic connection through the saddles - Vladaya (860 m), BukaPreslap (1090 m) and Yarema - $1195 \mathrm{~m}$. All this has a certain impact on the microclimatic conditions in the corresponding parts of the mountain. The dimensions of the mountain are about $23 \mathrm{~km}$ long and $19 \mathrm{~km}$ wide, its area is $27800 \mathrm{ha}\left(278 \mathrm{~km}^{2}\right)$ and represents $0.25 \%$ of the territory of Bulgaria. Highest peak is Cherni Vruh $2290 \mathrm{~m}$

Above 1000 species of scuttle flies are described from the Palaearctic Region, most of them - from Europe. In Bulgaria, 130 species are known up to now. The new taxa for Bulgaria are marked with one asterisk

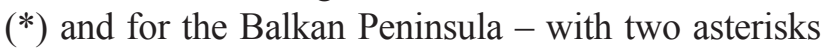
(**). All materials are deposited in the author's collection at the National Museum of Natural History, Bulgarian Academy of Sciences, Sofia.

\section{Material and methods}

The material has been collected by combining transects and stationary methods. Stationary methods were represented by different types of traps (Malaise trap, pitfall traps and traps in tree crowns) and entomological net. The traps are white conical plastic cups with a base diameter of $65 \mathrm{~mm}$, an opening of $88 \mathrm{~mm}$ and a height of $118 \mathrm{~mm} .4 \%$ formalin was used as a fixative with some detergent. Here we use the name "soil traps" for the pitfall traps and "tree trap" for those hanged on the branches of the trees. Part of the material was collected by MSS traps ("milieu souterrain superficiel", published already in Langourov et al., 2014).

\section{Collecting sites}

\section{- Northern part}

Deciduous forest belt (mixed and beech forests). Composed mainly of Corylus avellana L., Carpinus betulus L. and with participation of Quercus dalechampii Ten., Fagus sylvatica L., Betula pendula Roth, Sambucus nigra $\mathrm{L}$. The grass cover is composed mainly of different species of Poa, Festuca, Bromus, Phleum, as well 
as Anemone ranunculoides L., Allium ursinum L., Heleborus odorus W. et K., Corydalis sp., Erytronium dens-canis L.

[N1.01] above Simeonovo Suburb, $820 \mathrm{~m}$ a.s.1.

[N1.02] near Boyana Suburb, 850-870 m a.s.1.

[N1.03] above Boyana Suburb, $1080 \mathrm{~m}$ a.s.l.

[N1.04] above Boyana Lake, 1260 m a.s.l.

[N1.05] E of Kopitoto Peak, 1350 m a.s.1.

[N1.06] Kirova Livada Place, 1360 m a.s.l.

[N1.07] near Kamen Del Chalet, 1470 m a.s.l.

Spruce forest belt. The lower floor of these spruce forests is built mainly of Rubus idaeus L. and with the participation of a number of cereal grasses - Calamagrostis sp., Elymus sp. [N2.03]: Pinus peuce Griseb. forest with undergrowth of Arctostaphylos uva-ursi (L.) Spreng. and Vaccinium sp.

[N2.01] near Bor Chalet, $1600 \mathrm{~m}$ a.s.l.

[N2.02] near Tintyava Chalet, $1650 \mathrm{~m}$ a.s.l.

[N2.03] Gyoreva Cheshma Spring, 1750 m a.s.l.

Subalpine zone. Acidophilic psychrophytic grass associations - in these formations the largest areas are occupied by Nardus stricta L., Festuca valida (R. Uechtr.) Pénzes, Poa sp., Agrostis capillaris L. and Sesleria comosa Velen. [N3.04]: the vegetation is of hygropsychrophilic (peatbog) type, which is characterised by the dominance of Nardus stricta and the presence of a number of co-dominants - Potentila erecta Rausch., Carex spp., Deschampsia caespitosa (L.) P. Beauv., Sphagnum sp., with the participation of Veratrum lobelianum Bernh. and shrubs of Salix lapponum $\mathrm{L}$.

[N3.01] near Kamen Del Peak, 1840 m a.s.l.

[N3.02] Petrov Grob Place, $1850 \mathrm{~m}$ a.s.l., (with scattered trees of Pinus peuce)

[N3.03] Ushite Peak, 1906 m a.s.1.

[N3.04] Torfeno Branishte Reserve - Kapaklivets Place, 1860 m a.s.l.

- Eastern part

Beech forest belt. In this part of the mountain are located some of the best preserved beech forests. The beech forests in [E1.03] are highly enlightened and there is no undergrowth, as the grass cover is dominated by Luzula sp. In [E1.02] the co-dominant of beech is Alnus glutinosa (L.) Gaertn. In all other sites in the undergrowth Euonymus latifolius (L.) Mill is found which is dominant in the lower floor of [E1.04] and [E1.05], Rosa sp., Rubus sp. and single specimens of Sorbus aucuparia L., Viburnum opulus L., Crataegus monogyna Jacq., Corylus avellana. The grass cover is with the participation of Poa nemoralis L., Digitalis sp., Lunaria sp., and around the upper border of this belt there are Galium odoratum (L.) Scop. and Luzula sylvatica (Huds.) Gaudin.

[E1.01] Byala Voda Place near Yarema Saddle, $1130 \mathrm{~m}$ a.s.l.

[E1.02] near Gurgulitsa River, near Zheleznitsa Village, $1140 \mathrm{~m}$ a.s.l.

[E1.03] above Zheleznitsa Village, near Bukite Peak, $1200 \mathrm{~m}$ a.s.l.

[E1.04] above Bistritsa Village, $1200 \mathrm{~m}$ a.s.l.

[E1.05] near Bistritsa River, above Bistritsa Village, $1270 \mathrm{~m}$ a.s.l.

[E1.06] Bistrishko Branishte Reserve, near Bistritsa River, $1400 \mathrm{~m}$ a.s.l.

Spruce forest belt. Here are located one of the best preserved spruce forests within the mountain, for the protection of which was created one of the oldest nature reserves in Bulgaria and on the Balkans - "Bistrishko Branishte" (founded in 1934). The spruce forests in this part are centuries-old (over 150 years) monodominant, with the participation of single trees of Abies alba Mill. and Pinus sylvestris L., and the lower floor is mainly of Luzula sylvatica, Festuca sp., Geranium sp., Oxalis acetosella L. and scattered specimens of Rubus idaeus and Doronicum columnae Ten. Near the upper border of the belt the trees are thinning and the undergrowth is mainly of Juniperus communis ssp. alpina (Suter) Celak. and Vaccinium myrtillus L., which in the highest parts grow alone.

[E2.01] Bistrishko Branishte Reserve - Golyamata Gramada Place, 1550 m a.s.1.

[E2.02] Bistrishko Branishte Reserve $-\mathrm{N}$ of Golyamata Gramada Place, 1620 m a.s.l.

[E2.03] Bistrishko Branishte Reserve - Prisoite Place, $1750 \mathrm{~m}$ a.s.l.

Subalpine zone. Acidophilic psychrophytic grass associations - the largest areas are occupied by Festuca valida, Poa sp., Agrostis capillaris L., Bruckenthalia spiculifolia (Salisb.) Rchb., Vaccinium spp., Salix waldsteiniana Willd. and in [E3.02] is situated one of the largest communities of dwarf pine (Pinus mugo Turra) within the mountain.

[E3.01] Belcheva Skala Peak, 1831 m a.s.l. (limestone)

[E3.02] above Aleko Chalet, $1900 \mathrm{~m}$ a.s.1.

[E3.03] Bistrishko Branishte Reserve - Golyam Kupen Peak, 1930 m a.s.l.

[E3.04] near Malak Rezen Peak, 2150 m a.s.l. 
- Western part

Mixed forest belt. The locality is in an aspen forest (Populus tremula L.) with participation of Carpinus betulus, Corylus avellana, Betula pendula and Sambucus nigra. The grass cover is composed mainly of different species of Poa, Festuca, Bromus, Phleum, as well as Anemone ranunculoides, Allium ursinum, Heleborus odorus, Corydalis sp., Erytronium dens-canis.

[W1.01] near Matnitsa River, 1000 m a.s.1.

Spruce belt. The spruce forests in this part are sparse with participation of Fagus sylvatica and Betula pendula and the lower floor is mainly of Luzula sylvatica, Festuca sp., Rubus idaeus.

[W2.01] near Ostritsa Chalet, $1650 \mathrm{~m}$ a.s.1.

Subalpine zone. [W3.01]: acidophilic psychrophytic grass formations with the participation of Chamaecytisus sp., Luzula sp., Carex sp., J. communis ssp. alpina, Arctostaphylos uva-ursi and single specimens of Rubus idaeus. [W3.02] and [W3.03]: the vegetation is of hygropsychrophilic (peatbog) type, which is characterised by the dominance of Nardus stricta and the presence of a number of co-dominants Potentila erecta, different species of Carex, Sphagnum, with the participation of Luzula sp., Alchemilla sp. and shrubs of Salix lapponum L. and J. communis ssp. alpina. [W3.04-06] are characterised by the alpine appearance of the vegetation cover: N. stricta, Sesleria comosa, Festuca valida, F. nigrescens Lam., Poa media Schur., P. annua L., Agrostis capillaris.

[W3.01] Konyarnika Place, 1870 m a.s.1.

[W3.02] Choveshki Kosti Place, 1950 m a.s.l.

[W3.03] Torfeno Branishte Reserve - Tri Kladentsi Place, $2020 \mathrm{~m}$ a.s.1.

[W3.04] Selimitsa Peak, 2041 m a.s.l.

[W3.05] Torfeno Branishte Reserve - Samara Peak, $2108 \mathrm{~m}$ a.s.l.

[W3.06] near Vurla River, 2150 m a.s.1.

\section{- Southern part}

Mixed forest belt. The locality is composed mainly of Quercus frainetto Ten. and Quercus dalechampii with the participation of Crataegus monogyna, Carpinus betulus, Corylus avellana. The grass cover is mainly of different species of Poa, Festuca, Bromus, Phleum, as well as Anemone ranunculoides.

[S1.01] near Bosnek Village, 940 m a.s.1.

Beech forest belt. The beech forests in this part of the mountain have been heavily exploited in the past and currently consist of younger sparse beech trees with the participation of other tree and shrub species. Around the lower border of the belt there are Carpinus betulus, Corylus avellana, Cornus mas L., and around its upper border (which for this part is the upper border of the forest due to increased anthropogenic intervention in the past) - Juniperus communis ssp. alpina. Due to the low density of these forests, the grass cover is diverse, with a predominance of Poa nemoralis, Digitalis sp., Lunaria sp. and around the upper border are found Asperula sp., Luzula sylvatica.

[S2.01] Ribni Dol Place, 1240 m a.s.l.

Spruce belt. In this part of the mountain over 1600 $\mathrm{m}$ the forest has been almost completely destroyed, as in some places single coniferous trees and bushes have been preserved, and in the river valleys - Alnus viridis (Chaix) DC. with participation of Fagus sylvatica as the lower floor is mainly of Luzula sylvatica, Festuca sp., Rubus idaeus.

Subalpine zone. Acidophilic psychrophytic grass formations with the participation of a number of alpine elements - Sesleria comosa, Festuca valida, F. nigrescens, Poa media, Agrostis rupestris All., Juncus trifidus L., Alopecurus gerardii Vill., Crocus veluchensis Herb., Dianthus micropis Boiss.

[S4.01] Chernoto Plato Place, $2050 \mathrm{~m}$ a.s.1.

[S4.02] Yarlovski Kupen Peak, 2173 m a.s.l.

[S4.03] near Cherni Vruh Peak, 2200 m a.s.l.

[S4.04] Cherni Vruh Peak, 2280 m a.s.l.

\section{Results}

\section{Aenigmatiinae}

1. Aenigmatias franzi Schmitz, 1950 - Material examined: [E3.01] soil traps: 7.VIII-6.IX.1999, 1 $\hat{0} \cdot$ [W3.02] soil traps: 3.VII-7.VIII.1999, 2 ठో $\bullet$ [W3.03] soil traps: 3.VII-7.VIII.1999, 1 ठे. Palaearctic species, known from Europe (Austria, Switzerland, Czech Republic, Great Britain) and Asia (Mongolia). For the Balkan Peninsula reported from Bulgaria and Montenegro (Langourov, 2009). Other localities in Bulgaria: Central Stara Planina Mts and Pirin Mts (Langourov, 2009).

2. Aenigmatias lubbocki (Verrall, 1877) - Material examined: [S1.01] Malaise trap: 4-10.VIII.1998, 5

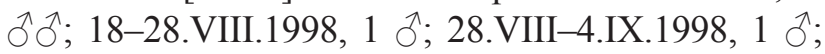
29.VII-10.VIII.1999, 4 $\widehat{\partial}, 2$ 우. Palaearctic species, known from Europe and Asia (China). Other localities 
in Bulgaria: Central Stara Planina Mts (Langourov, 2009).

Phorinae

** 3. Anevrina curvinervis (Becker, 1901) - Material examined: [N1.01] Malaise trap: 10-18.V.1997, 2 ○うં; 28.IV-1.V.1998, 1 §. Holarctic species, known from Middle and North Europe and North America. New region: Balkan Peninsula - Bulgaria.

4. Anevrina thoracica (Meigen, 1804) - Material examined: [N1.03]: 30.V.1993, 1 ô $\bullet$ [E1.05] soil traps:

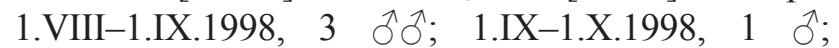
1.V-1.VI.1999, 1 q; 1.VIII-1.IX.1999, 5 ठึં; 1.IX-1.X.1999, 1 ते • [N1.05] Malaise trap: 19-26.VI.1998, 1 $\hat{\sigma} \cdot[\mathrm{N} 1.06]$ soil traps: 1.VII-1.VIII.1998, 1 ठิ; 1.VIII-1.IX.1998, 2 ठิ [E2.01]: 1.VIII-1.IX.1998, 4 ठิฎิ, soil traps; 1.VII-1.VIII.1999, 1 ᄋ, tree traps; 1.VIII-1.IX.1999, $1 \hat{\partial}$, tree traps; $1 \hat{\partial}$, soil traps - [N3.02]: 4.VII-6.VIII.1999, 4 ठㅊำ $\bullet$ [E3.02]: 1.VI-1.VII.1999, 10 , tree traps; 1.VII-1.VIII.1999, 1 ठ , soil traps • [W3.03] soil traps: 12.VI-3.VII.1999, 1 §. Holarctic; known from Europe and North America. Reported from Croatia (Langhoffer, 1919) and Montenegro (Langourov, 2004a). Other localities in Bulgaria: Osogovska Mt (Beschovski \& Langourov, 1997).

* 5. Anevrina unispinosa (Zetterstedt, 1860)-Material examined: [E1.05] soil traps: 1.VIII-1.IX.1998, $1 \hat{\delta} \cdot[\mathrm{N} 1.05]$ Malaise trap: 29.V-5.VI.1998, 1 $\widehat{\bullet}$ [N1.06] soil traps: 1.VI-1.VII.1999, 1 $\widehat{\jmath}$. PalaearcticOriental species, known from Europe, Asia (Japan) and Oriental Region (Myanmar). For the Balkan Peninsula reported from Montenegro (Langourov, 2004a).

6. Anevrina urbana (Meigen, 1830) - Material ex-

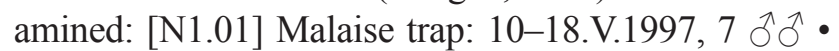
[S1.01] Malaise trap: 3-17.IV.1999, 1 के; 17-30.IV.1999, 3 $\widehat{\partial}$. Holarctic species, known from Europe, Asia (Western Siberia) and North America. For the Balkan Peninsula reported from Croatia (Becker, 1901). Other localities in Bulgaria: Sofia Plain and Western Rhodope Mts (Langourov, 2004b, 2010).

7. Borophaga irregularis (Wood, 1912) - Material examined: [E1.04]: 27.VIII.1992, 1 o $\bullet$ [E1.05] soil traps: 1.VIII-1.IX.1999, 1 त $\bullet \quad$ [N1.06]: 1.IX-1.X.1999, 3 ðð, soil traps; 1.IX-1.X.1999, 1 早, tree traps. European species. Published for the mountain by Beschovski \& Langourov (1997).

* 8. Chaetopleurophora bohemanni (Becker, 1901)

- Material examined: [N1.05] Malaise trap:
29.V-5.VI.1998, 1 Oे. European species, known for the Balkan Peninsula from Croatia (Schmitz, 1924).

9. Chaetopleurophora erythronota (Strobl, 1892)Material examined: [N1.01] Malaise trap:

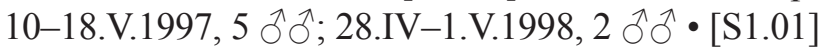
Malaise trap: 4-10.VIII.1998, 2 ठิ ठึ; 10-18.VIII.1998, 1 ภ̂, 1 ᄋ ; 28.VIII-4.IX.1998, 1 क; 25.IX-2.X.1998, 1 o - [N1.05] Malaise trap: 26.VI-3.VII.1998, 2 우우 between [N1.07] to [N2.01]: 28.VI.1993, 1 $\mathrm{O}^{\text {. }}$. Holarctic species, known from Europe, Asia (Russian Far East) and North America. Other localities in Bulgaria: South Black Sea Coast (Beschovski \& Langourov, 1997), Western Rhodope Mts (Langourov, 2010).

** 10. Chaetopleurophora spinosior Schmitz, 1938 - Material examined: [N1.06]: 1.V-1.VI.1998, $1 \hat{\partial}$, tree traps; 1.V-1.VI.1999, $1 \hat{\jmath}$, soil traps. European species, known from Middle Europe. New region: Balkan Peninsula - Bulgaria.

** 11. Chaetopleurophora spinosissima (Strobl, 1892) - Material examined: [N1.01] Malaise trap: 10-18.V.1997, 1 ․ Palaearctic species, known from Middle and North Europe and Asia (Russian Far East). New region: Balkan Peninsula - Bulgaria.

12. Conicera dauci (Meigen, 1830) - Material examined: [S1.01] Malaise trap: 18-28.VIII.1998, 3 $\widehat{\partial}$ - [N1.05] $1150 \mathrm{~m}: 21 . \mathrm{VII} .1992,1$ ๙ $\bullet$ [N1.05] Malaise trap: 19-26.VI.1998, 1 ふં; 26.VI-3.VII.1998, 5 ふぇં. Holarctic species, known from Europe, Macaronesia, Asia (Russian Far East, Japan, China) and North America. For the Balkan Peninsula reported also from Montenegro (Langourov, 2004a). Other localities in Bulgaria: Sofia Plain (Langourov, 2004b).

** 13. Conicera floricola Schmitz, 1938 - Material examined: [S1.01] Malaise trap: 11-25.IX.1998, 1 ภे; 25.IX-2.X.1998, $1 \hat{\sigma} \cdot$ [E1.02]: 15.IV.2000, $1 \hat{\delta}$, on flowers of Petasites hybridus ochroleucus (Boiss. et Huet) Sourek • [E1.03]: 15.IV.2000, 1 ठ • [N1.05] Malaise trap: 29.V-5.VI.1998, 2 ô $\hat{0} ;$ 5-12.VI.1998, 2

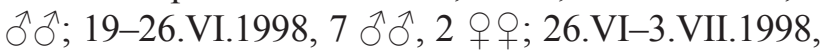
$4 \hat{\jmath} \hat{\delta}$. Palaearctic species, known from Europe and Asia (Israel, Russian Far East). New region: Balkan Peninsula-Bulgaria.

** 14. Conicera schnittmanni Schmitz, 1926 - Material examined: [N1.05] Malaise trap: 3-8.VII.1998, 1 ${ }^{3}$. Palaearctic; known from Europe and Asia (Russian Far East). New region: Balkan Peninsula - Bulgaria.

* 15. Conicera similis (Haliday, 1833) - Material examined: [S1.01] Malaise trap: 4-10.VIII.1998, 25

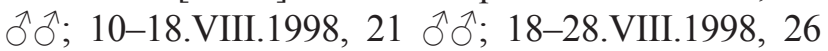

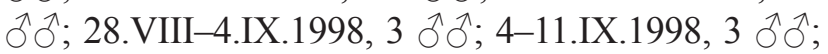




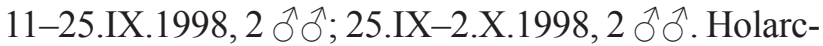
tic species, known from Europe, North Africa (Algeria, Egypt), Asia (Turkey, Israel) and North America. For the Balkan Peninsula reported from Croatia (Pax, 1937; Schmitz, 1938).

** 16. Conicera tibialis Schmitz, 1925 - Material examined: [S1.01] Malaise trap: 4-10.VIII.1998, 2

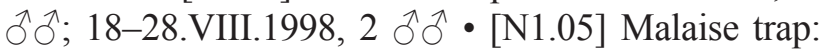

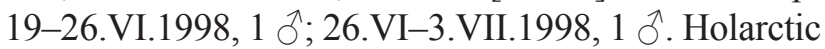
species, known from Europe, North Africa (Egypt), Macaronesia, Asia (Israel, Iran, Tajikistan, Russian Far East) and North America. New region: Balkan Peninsula - Bulgaria.

* 17. Diplonevra amphichaeta (Schmitz, 1949) Material examined: [E1.04] $1000 \mathrm{~m}$, leg. V. Beschovski: 18.VI.1972, 1 ठै. European species, known from Austria and Slovenia (Schmitz, 1953: "Krain: Loitsch" = Logatec). In Beschovski \& Langourov, 1997 wrongly determined as Diplonevra lophochaeta (Schmitz, 1927).

18. Diplonevra crassicornis (Meigen, 1830) - Material examined: [N1.05] Malaise trap: 5-12.VI.1998, 3

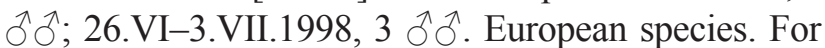
the Balkan Peninsula reported from Bosnia-Herzegovina (Strobl, 1898) and Croatia (Langhoffer, 1919). Other localities in Bulgaria: Sofia Plain (Langourov, 2004b).

19. Diplonevra florescens (Turton, 1801) - Material examined: [N1.01] Malaise trap: 10-18.V.1997, 5

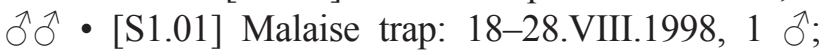
28.VIII-4.IX.1998, 1 J; 900 m, near Bosnek Village, near Popov Izvor Karst spring, MSS traps: 6.VI-2.X.2013, 8 ○うં; 1120 m, above Bosnek Village, on the road to Chuipetlyovo, scree in mixed wood, MSS traps: 6.VI-2.X.2013, 1 - $\cdot[\mathrm{N} 1.05]$ Malaise trap: 29.V-5.VI.1998, 1 ภे; 5-12.VI.1998, 1 ภे. Palaearctic; known from Europe and Asia (Armenia, Georgia, Turkey, China, Russian Far East). For the Balkan Peninsula reported from Italy (Schmitz, 1928). Other localities in Bulgaria: Sofia Plain (Beschovski \& Langourov, 1997), W Rhodope Mts (Langourov, 2010) and southern part of Vitosha Mt (Langourov et al., 2014).

20. Diplonevra funebris (Meigen, 1830) - Material examined: [S1.01] Malaise trap: 4-10.VIII.1998, 16

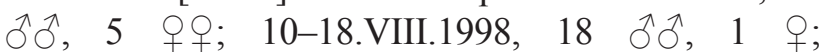
18-28.VIII.1998, 32 ○ึ 1 ๆ; 28.VIII-4.IX.1998, 10 ठิ 25.IX-2.X.1998, 2 ô ô; 2-31.X.1998, 1 ô, 1 ᄋ; 954 $\mathrm{m}$, near Bosnek Village, near Academik Cave, MSS traps: 6.VI-2.X.2013, $2 \hat{\jmath} \widehat{\partial}, 4$ 우 • above Bosnek Vil- lage, $1200 \mathrm{~m}$, oak wood, soil traps (leg. G. Tsonev): 30.VII-18.IX.1994, $110 \hat{0}$ [S2.01]:

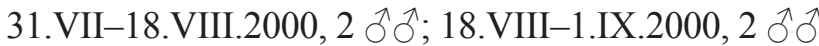
- [N1.06] soil traps: 1.V-1.VI.1998, 1 Oे. Holarctic; known from Europe, North Africa (Algeria, Egypt), Macaronesia, Asia (Israel, Iran, China, Russian Far East) and North America. For the Balkan Peninsula reported from Croatia (Pax, 1937) and Greece (Disney, 1991). Other localities in Bulgaria: Danube Plain, Lozen Mt (Beschovski \& Langourov, 1997), Sofia Plain (Langourov, 2004b), Eastern Rhodope Mts (Langourov, 2004c) and southern part of Vitosha Mt (Langourov et al., 2014).

21. Diplonevra glabra (Schmitz, 1927) - Material examined: [N1.01] Malaise trap: 10-18.V.1997, 21

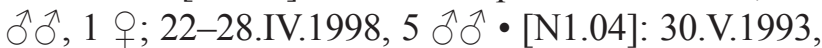

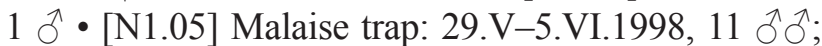

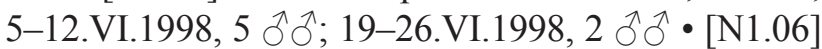
soil traps: 1.VIII-1.IX.1999, 3 우. European species. Other localities in Bulgaria: Sofia Plain (Langourov, 2004b).

22. Diplonevra nitidula (Meigen, 1830) - Material examined: [S1.01] Malaise trap: 4-10.VIII.1998, 16

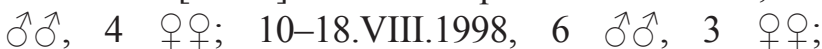
18-28.VIII.1998, 17 ふึે, 5 o

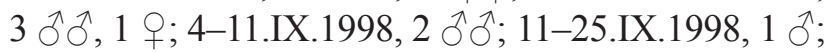
25.IX-2.X.1998, 4 ठิठี; 2-31.X.1998, 11 ठิठે • [N1.05] Malaise trap: 29.V-5.VI.1998, 1 ऊิ;

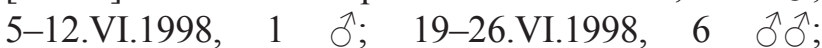

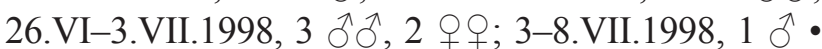
[N1.06] soil traps: 1.VII-1.VIII.1998, 1 के; 1.VI-1.VII.1999, 1 ô. Holarctic species, known from Europe and North America. For the Balkan Peninsula reported from Greece (Schmitz, 1928). Other localities in Bulgaria: Sofia Plain and Ograzhden Mt (Beschovski \& Langourov, 1997).

** 23. Gymnoptera vitripennis (Meigen, 1830) Material examined: [S1.01] Malaise trap: 18-28.VIII.1998, 1 के $\bullet$ [N1.06] soil traps: 1.VII-1.VIII.1998, 1 गे. European species, known from Middle Europe. New region: Balkan Peninsula Bulgaria.

** 24. Peromitra carinifrons (Zetterstedt, 1848) Material examined: [N1.06] soil traps: 1.VIII-1.IX.1999, 1 ô. Palaearctic species, known from Middle and North Europe, Asia (Mongolia and Russian Far East). New region: Balkan Peninsula Bulgaria.

** 25. Peromitra incrassata (Meigen, 1830) - Material examined: [N1.02] Malaise trap: 
24.VIII-1.IX.1999, 1 $\hat{0} \cdot$ [S1.01] Malaise trap: 18-28.VIII.1998, 1 o • [E1.05]: 1.VIII-1.IX.1999, 4 $\widehat{\partial} \hat{\partial}$, soil traps; $2 \hat{\partial}, 1$ ㅇ, tree traps. European species. New region: Balkan Peninsula - Bulgaria.

26. Phora atra (Meigen, 1804) - Material examined: [N1.01] Malaise trap: 10-18.V.1997, 3 ठ๙ [S1.01] Malaise trap: 10-18.VIII.1998, 2 ठึ่;

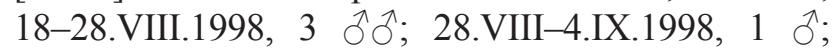
11-25.IX.1998, 1 ठં; 17-30.IV.1999, 1 ๙ •[W1.01]

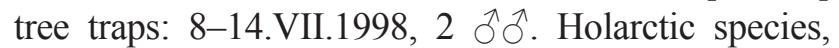
known from Europe, North Africa (Algeria), Macaronesia, Asia (Israel) and North America. For the Balkan Peninsula reported from Croatia (Langhoffer, 1919) and North Macedonia (Langourov, 1999). Other localities in Bulgaria: Sofia Plain (Langourov, 2004b) and Eastern Rhodope Mts (Langourov, 2004c).

** 27. Phora dubia (Zetterstedt, 1848) - Material examined: [N1.01] Malaise trap: 10-18.V.1997, 1 o [E1.05]: 1.V-1.VI.1998, 1 ภ, 23 $q$ q , tree traps; 1 ô, 5 우, soil traps; 1.VII-1.VIII.1998, 1 , tree traps; 1.XI.1998-1.V.1999, 9 우, tree traps; 1.V-1.VI.1999, 11 우, tree traps; 1 ô, soil traps $\cdot[\mathrm{N} 1.06]$ soil traps: 1.V-1.VI.1998, 1 ㅇ ; 1.V-1.VI.1999, 1 ○ 1500 m, E of Septemvri Chalet, beech wood, tree traps (leg. T. Ljubomirov): 3-10.VII.1998, 1 우 $1535-1850 \mathrm{~m}$, between Fonfon Chalet to Konyarnika Place, spruce wood: 26.V.1996, 1 ठै - [E2.01]: 30.V.1993, 1 क;

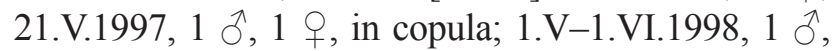
3 우, tree traps; 1.VIII-1.IX.1998, 1 ठ, soil traps; 8.V.1999, 1 đ; 1.V-1.VI.1999, 1 ๙ , 5 우, tree traps •

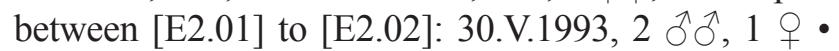
between [E2.02] to [E2.03]: 30.V.1993, 1 ô, 2 우우 21.V.1997, 1 o, 1 O , in copula • [N2.02]: 30.V.1993, 2

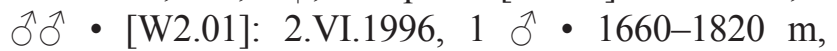
between Ostritsa to Edelvais Chalet, spruce wood:

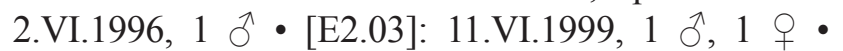
[W3.01]: 2.VI.1996, 1 o • [E3.02]: 1.V-1.VI.1999, 3

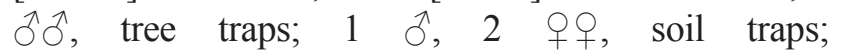
1.VII-1.VIII.1999, 1 q, soil traps $-2100 \mathrm{~m}$, near Golyam Rezen Peak, subalpine zone (leg. E. Manasieva): 4.VI.1995, 1 §. Palaearctic species, known from Europe and Asia (Japan). New region: Balkan Peninsula-Bulgaria.

28. Phora edentata Schmitz, 1920 - Material examined: [S1.01] Malaise trap: 4-10.VIII.1998, 1 ते;

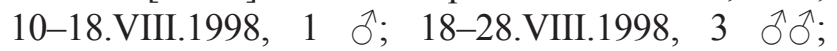

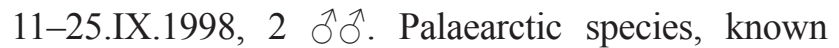
from Europe and Asia (Russian Far East, Japan, China). For the Balkan Peninsula reported from Croatia (Schmitz, 1955). Other localities in Bulgaria: Sofia
Plain (Langourov, 2004b) and Eastern Rhodope Mts (Langourov, 2004c).

29. Phora hamata Schmitz, 1927 - Material examined: [N1.01] Malaise trap: 10-18.V.1997, 3 ठึ 12-17.V.1998, $1 \hat{\boldsymbol{O}} \cdot$ between [E1.05] to [E1.06]: 27.VIII.1992, 1 ठิ. European species, known from Middle Europe. Other localities in Bulgaria: Eastern Rhodope Mts (Langourov, 2004c).

30. Phora holosericea Schmitz, 1920 - Material examined: [S1.01] Malaise trap: 10-18.VIII.1998, 1 ๙ - [N1.05] Malaise trap: 3-8.VII.1998, 1 స̂. Holarctic species, known from Europe, Asia (Israel, Iran, Kyrgyzstan, Mongolia, China, South Korea, Japan, Russian Far East) and North America. For the Balkan Peninsula reported from Greece (Disney, 1991) and North Macedonia (Langourov, 1999). Other localities in Bulgaria: Sofia Plain (Langourov, 2004b) and Western Rhodope Mts (Langourov, 2010).

** 31. Phora horrida Schmitz, 1920 - Material examined: [E3.02], tree traps: 1.V-1.VI.1999, 1 ô, 1 ㅇ․ European high mountain species, known from Middle Europe (Alps, Tatra Mts and Carpathian Mts). New region: Balkan Peninsula - Bulgaria.

32. Phora penicillata Schmitz, 1920 - Material examined: [N1.07]: 31.V.1998, 1 o $\bullet$ between [E2.01] to [E2.03]: 20.VIII.1992, $1 \hat{\sigma} \cdot[$ [E3.02], soil traps: 1.VII-1.VIII.1999, 1 ภ, 1 q $\bullet$ [S4.01]: 6.VI-5.VII.1999, 1 ô. European species. Other localities in Bulgaria: W Rhodope Mts (Langourov, 2010).

* 33. Phora pubipes Schmitz, 1920 - Material examined: [E1.04]: 27.VIII.1992, 1 ते • [E1.05]: 1.VIII-1.IX.1998, 1 ठ, 1 q, tree traps; 1.VIII-1.IX.1999, 1 ภ, 1 ㅇ, tree traps; 1 ภ, 3 우, soil traps $1500 \mathrm{~m}, \mathrm{E}$ of Septemvri Chalet, beech wood, tree traps: 3-10.VII.1998, 1 o $\bullet$ between [E2.01] to [E2.03]: 20.VIII.1992, 2 ते $\quad$ [E2.01]:

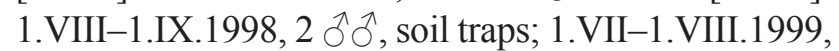

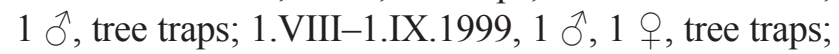
$1 \hat{\partial}$, soil traps. Palaearctic-Oriental species, known from Europe, Asia (Mongolia, Japan) and Oriental Region. For the Balkan Peninsula reported from BosniaHerzegovina (Strobl, 1898 as Trineura aterrima).

34. Phora stictica Meigen, 1830 - Material examined: 1260-1360 m, near Esperanto (Zeleni Patruli) Chalet, mixed wood: 3.IX.1992, 1 त $\cdot$ [E1.05], tree traps: 1.VIII-1.IX.1999, $1 \hat{\sigma} \cdot$ between [E1.05] to [E1.06]: 27.VIII.1992, 1 ภ • [E1.06]: 20.VIII.1992, 1 $\hat{\sigma} \cdot$ between [E1.06] to [E2.01]: 3.IX.1992, 1 त [N1.07]: 20.VIII.1992, 1 今 $\bullet$ [E2.01]: 1.VIII-1.IX.1998, 4 ठึ ठૈ, soil traps; 1.VII-1.VIII.1999, 
2 ふふ, 1 q, tree traps; 1 ก, soil traps;

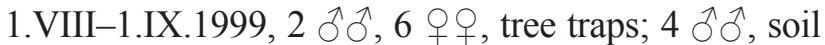
traps $\bullet$ between [E2.01] to [E2.02]: 27.VIII.1992, 1 \%; 3.IX.1992, 1 ○ $\cdot[\mathrm{N} 2.01]: 21 . V I I .1992,1 \AA$, on Rubus sp. leaf • [N2.03]: 20.VII.1992, 1 ก $1840 \mathrm{~m}$, near Aleko Chalet, spruce wood (leg. V. Beschovski): 10.VIII.1984, 1 o $\bullet$ [E3.02], tree traps:

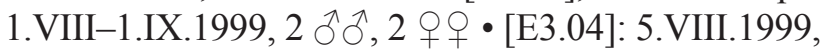
$7 \hat{\partial} \hat{\partial}, 1$, , on racemes of $A$. sylvestris L. HolarcticNeotropical; known from Europe, North America and South America (withouth southernmost part). Reported from Croatia (Schmitz, 1955) and North Macedonia (Langourov, 1999). Other localities in Bulgaria: W Rhodope Mts (Langourov, 2010).

35. Phora tincta Schmitz, 1920 - Material examined: [N1.01] Malaise trap: 22-28.IV.1998, 1 ○ [N1.05] 1150 m: 21.VII.1992, 2 ठे 0 [E1.05]:

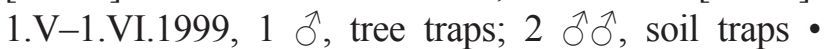
between [E1.05] to [E1.06]: 20.VIII.1992, 1 ô $\bullet$ [N1.06]: 20.VIII.1992, 2 ठో soil traps - [E1.06]: 11.VI.1999, 2 ठेंग, on Rubus idaeus leafs - [E2.01]: 1.VII-1.VIII.1998, 2 ठ $\sigma^{\lambda}$, tree traps; 1.VIII-1.IX.1998, 3 ठิへ, soil traps;

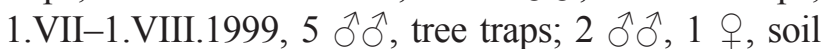
traps $\bullet$ between [E2.01] to [E2.02]: 28.VI.1993, 4 ठえ $\widehat{\bullet}$ [N2.01]: 21.VII.1992, 1 त, on flowers of Anthriscus nitida (Wahlenb.) Garcke • between [E2.02] to [E2.03]:

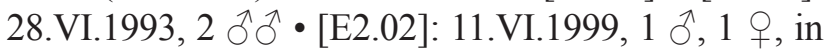
copula - on Rubus idaeus leaf. Palaearctic species, known from Europe and Asia (Israel, West Siberia, Tajikistan). For the Balkan Peninsula reported from Croatia (Schmitz, 1924) Slovenia (Schmitz, 1928) and Montenegro (Langourov, 2004a). Other localities in Bulgaria: Western Rhodope Mts (Langourov, 2010).

36. Spiniphora bergenstammi (Mik, 1864) - Material examined: [N1.02] Malaise trap: 1-18.IX.1999, 1

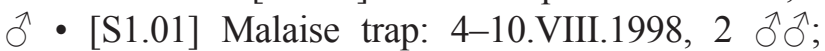

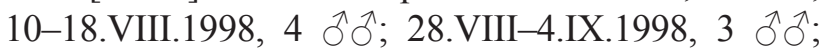
4-11.IX.1998, 1 of. Cosmopolitan species. For the Balkan Peninsula reported from Italy, Croatia, Greece (Schmitz, 1928) and North Macedonia (Langourov, 1999). Other localities in Bulgaria: Sofia Plain (Beschovski \& Langourov, 1997).

* 37. Spiniphora dorsalis (Becker, 1901) - Material examined: [N1.06], soil traps: 1.IX-1.X.1998,

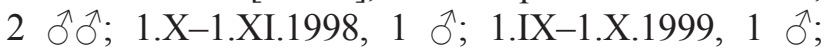
1.X-1.XI.1999, 1 ภิ • [N3.02]: 6.VIII-8.IX.1999, 1 ภิ. European species. For the Balkan Peninsula reported from Croatia (Becker, 1901) and North Macedonia (Langourov, 1999).
* 38. Spiniphora jugorum (Schmitz, 1924) - Material examined: [E2.01]: 1.VII-1.VIII.1999, 1 ô, tree traps - [E3.02], tree traps: 1.VIII-1.IX.1998, 1 ○; 1.V-1.VI.1999, 1 ふ; 1.VII-1.VIII.1999, 2 ふふ; 1.VIII1.IX.1999, 1 - [E3.04]: 5.VIII.1999, 1 क , on racemes of Angelica sylvestris L. - [E3.04] soil traps:

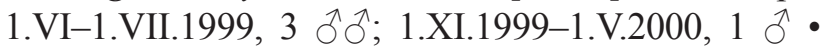

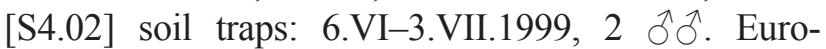
Caucasian species, known from the Alps, Tatra Mts, Durmitor Mt and Caucasus. For the Balkan Peninsula reported from Montenegro (Schmitz, 1928).

39. Spiniphora maculata (Meigen, 1830) - Material examined: [N1.01] Malaise trap: 22-28.IV.1998, 1

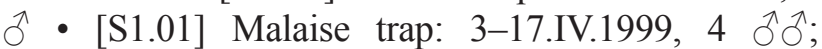
17-30.IV.1999, 2 ऊิरे - [N1.06], tree traps: 15.IV-1.V.1999, 1 ते $\quad$ [E3.04] soil traps:

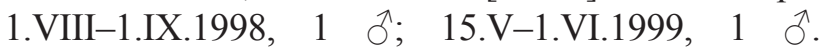
European; for the Balkan Peninsula reported from Croatia (Strobl, 1902) and Italy (Schmitz, 1928). In Bulgaria: E Rhodope Mts (Langourov, 2004c).

40. Triphleba antricola (Schmitz, 1918) - Material examined: [S1.01] Malaise trap: 11-25.IX.1998, 1 웅

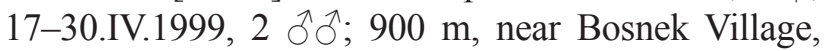
near Popov Izvor Karst spring, MSS traps: 6.VI-2.X.2013, 3 우; $910 \mathrm{~m}$, near Bosnek Village, Popov Izvor Cave, soil traps: 2.X-2.XI.2013, 8 $\widehat{\partial}, 31$ o o ; $1006 \mathrm{~m}$, near Bosnek Village, near Duhlata Cave, MSS traps: 6.VI-2.X.2013, 1 ○े; 1118 m, near Bosnek Village, Zhivata Voda Cave, soil traps:

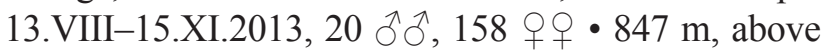
Boyana, Boyanski Kamak place, microcave, MSS trap: 30.IV-3.VI.2006, 8 우; [N1.06]: 1.IX-1.X.1998, 1 ô, soil traps; 4.XI.1998, 1 , among fallen leaves. Southeuropean species, widespread in the country. For the Balkan Peninsula also known from Bosnia and Herzegovina (Bezzi, 1911; Schmitz, 1919), Italy, Slovenia, Croatia, Serbia (Schmitz, 1919), Greece and Romania (Langourov, 2001). For the mountain reported also from Duhlata Cave (Hazelton, 1970).

41. Triphleba autumnalis (Becker, 1901) - Material examined: [E1.05]: 17.X.1992, 1 ठै; 1.XI.1998-1.V.1999, 1 Ô, soil traps; 1.X-1.XI.1999,

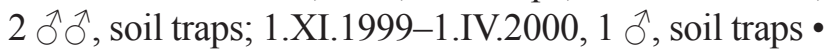
[N1.06]: 1.IX-1.X.1998, 2 ๙ิ ô, soil traps; 4.XI.1998, 1 $\hat{\partial}$, among fallen leaves; 1.XI.1998-15.IV.1999, 1 ㅇ,

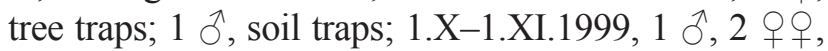
soil traps; 1.XI.1999-1.IV.2000, $4 \hat{\jmath} \widehat{\partial}, 1$ ㅇ, soil traps • [N1.06]: 2.X.1999, 1 o [E2.01], soil traps: 1.X-1.XI.1999, 1 ठ̊. European species. Other localities in Bulgaria: Eastern Rhodope Mts (Langourov, 2004c). 
42. Triphleba bicornuta (Strobl, 1910) - Material examined: [S1.01] Malaise trap: 25.IX-2.X.1998, 1 ๙ิ, 1 o; 2-31.X.1998, 8 ๙ิ - between [E1.05] to [E1.06]: 27.VIII.1992, 1 ○ [N1.06]: 1.X-1.XI.1998, 1 §, 1 q,

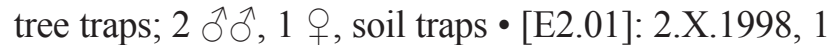

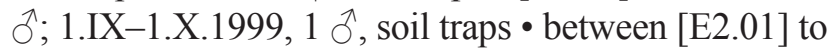
[E2.02]: 3.IX.1992, $1 \hat{\jmath} \cdot[E 2.02]: 17 . X .1992,1 \hat{\jmath} \bullet$ [E3.02], soil traps: 1.VIII-1.IX.1998, 1 ぶ;

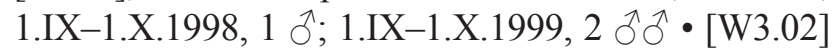

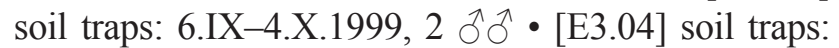
1.VIII-1.IX.1998, 2 ठぇં; 1.IX-1.X.1998, 3 ふぇ;

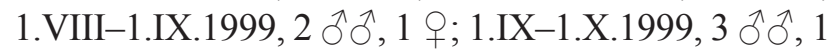
q • [S4.03]: 6.VIII-8.IX.1999, 1 ․ European species, known from North Europe and the mountains of Middle Europe. Other localities in Bulgaria: Rila Mts (Beschovski \& Langourov, 1997).

** 43. Triphleba citreiformis (Becker, 1901) - Material examined: [N1.05] Malaise trap: 5-12.VI.1998, 1 q. European species. New region: Balkan Peninsula - Bulgaria.

44. Triphleba distinguenda (Strobl, 1892) - Material examined: [N1.01] Malaise trap: 10-18.V.1997, 46

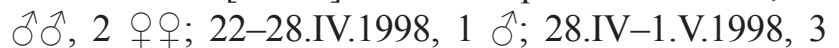
ઈิ 2 오; 10-18.VIII.1998, 3 ठึす; 18-28.VIII.1998, 3

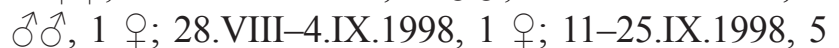

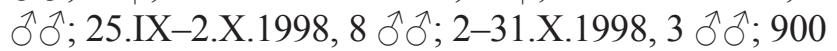
$\mathrm{m}$, near Bosnek Village, near Popov Izvor Karst spring, MSS traps: 6.VI-2.X.2013, 4 ठิす, 9 우 • - [N1.03]: 28.VI.1993, 1 o $1260-1360$ m, near Esperanto Chalet, mixed wood: 27.VIII.1992, 1 • [N1.05] Malaise trap: 29.V-5.VI.1998, 1 ๙; 19-26.VI.1998, 2 ठ઼ - [N1.06]: 3.IX.1992, 1 o; 1.VIII-1.IX.1998, 1 ㅇ, soil traps; 1.V-1.VI.1999, 1 , , soil traps; 1.IX-1.X.1999, 1 O , soil traps - between [N1.06] to [N1.07]: 27.VIII.1992, 1 ○ [E1.06]: 20.VIII.1992, $1 \hat{\jmath} \bullet$ between [N1.07] to [N2.01]: 28.VI.1993, 1 ふ.. Palaearctic species, known from Europe and Asia (Russian Far East). For the Balkan Peninsula also known from Montenegro (Langourov, 2004a). Other localities in Bulgaria: Sofia Plain and Vitosha Mountain (Beschovski \& Langourov, 1997; Langourov et al., 2014).

45. Triphleba dudai (Schmitz, 1918) - Material examined: between [N1.03] to [N1.04]: 30.V.1993, 1 ๙ 1260-1360 m, near Esperanto Chalet, mixed wood: 27.VIII.1992, 1 - $\quad[\mathrm{N} 1.05]$ Malaise trap: 26.VI-3.VII.1998, 1 ๙̋. European species. Published for the mountain by Beschovski \& Langourov (1997).

46. Triphleba gracilis (Wood, 1907) - Material examined: [N1.01] Malaise trap: 10-18.V.1997, 1 $ぇ$
[E2.01], tree traps: 1.IX-1.X.1998, 1 \%; 1.VIII-1.IX.1999, 1 ㄱ, 2 우우 between [E2.01] to [E2.02]: 27.VIII.1992, 1 ๑ [E2.02]: 3.IX.1992, 1 ○ • between [E2.02] to [E2.03]: 17.X.1992, 1 ○ • [E2.03]: 3.IX.1992, 1 ô. European species. Published for the mountain by Beschovski \& Langourov (1997).

47. Triphleba hyalinata (Meigen, 1830) - Material examined: [N1.02]: 27.X.1999, 1 o • [E1.05]: 1.IX-1.X.1998, 1 q, tree traps; 1.X-1.XI.1998, 1 \%, tree traps; 1.IX-1.X.1999, 2 qo, tree traps; 1.X-1.XI.1999, 1 \%, tree traps; 1 q, soil traps; 964 m, near Bosnek Village, near Duhlata Cave, MSS trap: 18.IV-16.VI.2007. European species. For the Balkan Peninsula also known from Italy (Funk \& Graffe, 1895). Other localities in Bulgaria: Central Stara Planina Mts, Enina Village; Sofia Plain, Sofia and Vitosha Mt (Langourov, 2001; Langourov et al., 2014); Eastern Rhodope Mts (Langourov, 2004c).

** 48. Triphleba hypopygialis (Schmitz, 1918) Material examined: [E2.01]: 1.IX-1.X.1998, 1 o, tree traps; 1.VII-1.VIII.1999, 1 त, 1 o, tree traps; 1.VIII-1.IX.1999, 1 ๆ, soil traps • [E3.02], soil traps: 1.VIII-1.IX.1999, 1 స. European species, known till now from Austria, Germany and France. New region: Balkan Peninsula - Bulgaria.

49. Triphleba inaequalis Schmitz, 1943 - Material examined: [E1.04]: 17.X.1992, 1 ふै • [E1.05], tree traps: 1.IX-1.X.1998, 1 ふ̋; 1.XI.1998-1.V.1999, 1 ふ;; 1.IX-1.X.1999, 1 §;; 900 m, near Bosnek Village, near Popov Izvor Karst spring, MSS traps: 2.X-2.XI.2013, $4 \hat{\partial} \hat{\sigma}$. European species. Reported also for the southern part of Vitosha Mt (Langourov et al., 2014).

50. Triphleba intermedia (Malloch, 1908) - Material examined: [S1.01] Malaise trap: 3-17.IV.1999, 3 ふै’ $1100 \mathrm{~m}$, above Zheleznitsa Village, mixed wood (leg. V. Beschovski): 18.V.1982, 5 ふ̄ર̂,, 1 ○ • [E1.03]: 15.IV.2000, 1 ๙ $1830 \mathrm{~m}$, Bistrishko Branishte Reserve, near Fizkulturnik Chalet, subalpine zone (leg. V.

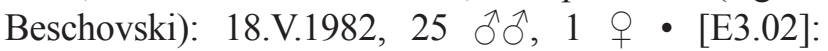
27.IV.2000, 1 o [E3.02], tree traps: 1.V-1.VI.1999, 1 ふ̄, 1 ○ [N3.03] soil traps: 29.V-4.VII.1999, 1 • [W3.04] soil traps: 5.VI-3.VII.1999, 1 ○ [E3.04], on snow: 27.IV.2000, 1 o $[$ [E3.04] soil traps:

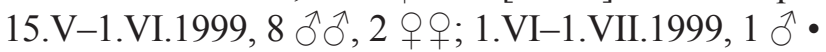

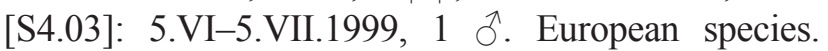
Other localities in Bulgaria: Eastern Rhodope Mts (Langourov, 2004b) and Western Rhodope Mts (Langourov, 2010).

** 51. Triphleba longifurcata (Schmitz, 1922) Material examined: [N1.06], soil traps: 
1.VIII-1.IX.1998, 1 ․ European species, known till now only from the type-locality in Hungary. New region: Balkan Peninsula - Bulgaria.

* 52. Triphleba lugubris (Meigen, 1830) - Material examined: [S1.01] Malaise trap: 25.IX-2.X.1998, 1 ठ - [E1.05] soil traps: 1.VIII-1.IX.1998, 1 ð. HolarcticOriental; known from Europe, North America and Oriental Region. On the Balkan Peninsula - Croatia (Pax, 1937 as Diploneura [sic!] lugubris (Meigen).

53. Triphleba nudipalpis (Becker, 1901) - Material examined: [N1.01] Malaise trap: 10-18.V.1997, 21

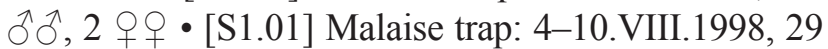

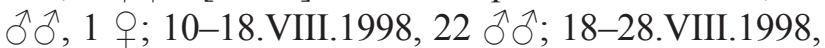

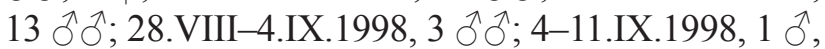

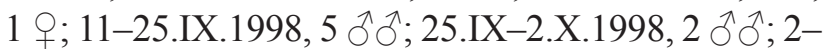
31.X.1998, 3 $\widehat{\jmath}, 1$ ㅇ. European species. Other localities in Bulgaria: Kresna Gorge (Langourov \& Sakalian, 2001).

54. Triphleba opaca (Meigen, 1830) - Material examined: [S1.01] Malaise trap: 3-17.IV.1999, 1 • [E1.05]: 1.XI.1998-1.V.1999, 1 क, soil traps; 1.XI.1999-1.IV.2000, 1 ô, tree traps • [N1.06]: 1.XI.1998-15.IV.1999, 1 गे, tree traps; 15.IV-1.V.1999, 1 Oे, 1 क , tree traps; 1 , soil traps;

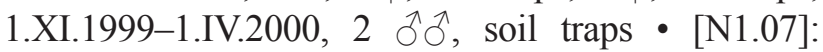
19.IV.1993, 1 ค • [E2.03]: 30.V.1993, 1 ठิ • [E3.02]: 1.V-1.VI.1998, 1 ㅇ, soil traps; 1.X.1998-1.V.1999, 1

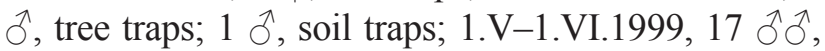

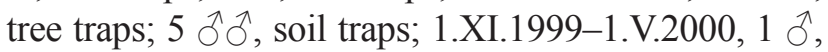
soil traps • [W3.02] soil traps: 5.VI-3.VII.1999, 3 우 $\bullet$ [W3.05] soil traps: 5.VI-3.VII.1999, 2 우 • [W3.04] soil traps: 5.VI-3.VII.1999, 1 ๆ [E3.04], on snow:

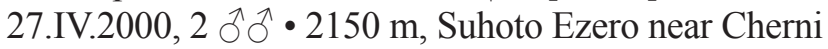
Vruh, subalpine zone, on snow (leg. E. Manasieva):

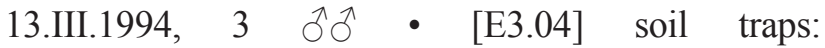

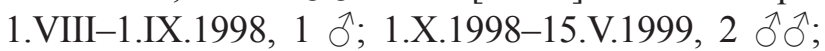
15.V-1.VI.1999, 9 ふぇึ, 1 ๆ • 2191 m, Malak Rezen,

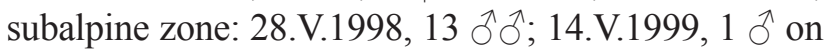
snow - $2280 \mathrm{~m}$, Cherni Vruh, subalpine zone: 26.V.1996, 1 §. Palaearctic; known from Europe and Asia (W Siberia and Russian Far East). For the Balkan Peninsula known from Italy (Funk \& Graffe, 1895), Albania and former Yugoslavia (Disney, 1991).

** 55. Triphleba pachyneurella (Schmitz, 1919) Material examined: [E2.02]: 28.V.1998, 1 तै [E3.02]: 1.X.1998-1.V.1999, 1 ô, soil traps; 1.V-1.VI.1999, $2 \AA \widehat{\jmath}$, tree traps $\bullet$ [E3.04] soil traps: 15.V-1.VI.1999, 1 o $-2280 \mathrm{~m}$, Cherni Vruh Peak, subalpine zone (leg. V. Beschovski): 18.V.1982, $1 \hat{\partial}$, on flower of Crocus veluchensis Herb. European species, known till now from Austria, Italy, Slovakia, Sweden and Finland. New region: Balkan Peninsula - Bulgaria.

56. Triphleba papillata (Wingate, 1906) - Material

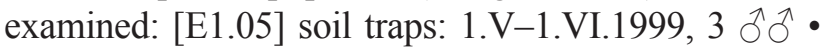
[N1.05] Malaise trap: 29.V-5.VI.1998, 3 ठิठ • [N1.06]: 1.XI.1998-15.IV.1999, 1 ô, 1 क, tree traps; 1.V-1.VI.1999, 2 ふึે, 2 qo+, soil traps - [E2.02]: 28.V.1998, 1 ơ; 964 m, near Bosnek, near Duhlata Cave, MSS trap: 18.IV-16.VI.2007. European; in Bulgaria: Eastern Rhodope Mts (Langourov, 2004b) and southern parts of Vitosha Mt (Langourov et al., 2014).

57. Triphleba trinervis (Becker, 1901) - Material examined: [S2.01]: 8-31.X.2000, 1 o • [E1.05]:

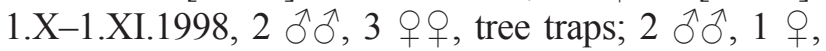

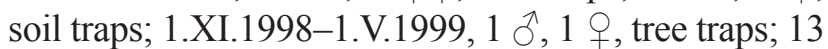

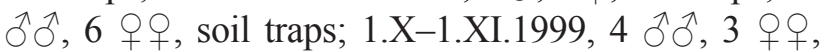
tree traps; 3 ठิग, 10 9 ,

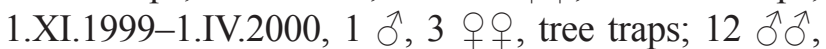

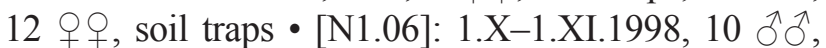
13 우우, tree traps; $7 \hat{\jmath} \widehat{\partial}, 17$ 우, soil traps; 4.XI.1998, 1 ò, 2 $q 9$, among fallen leaves;

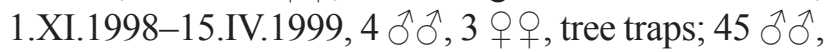
32 우, soil traps; 1.X-1.XI.1999, 2 ổ, 3 우, tree

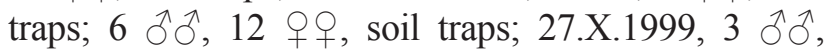
3 우, numerous among fallen leaves;

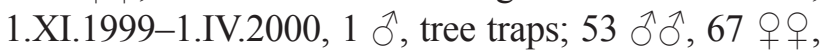
one pair in copula, soil traps • [E2.01]: 1.X-1.XI.1998, $1 \hat{\partial}$, tree traps; 1 गे, 1, , soil traps; 1.XI.1998-1.V.1999, 1 q, soil traps; 1.X-1.XI.1999,

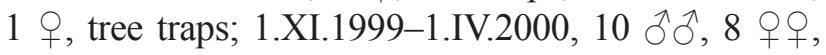
soil traps $1590 \mathrm{~m}$, near Sredets Chalet, spruce wood: 4.XI.1998, 1 ๆ • [E3.02]: 1.X.1998-1.V.1999, 1 Oे, soil traps; 1.X-1.XI.1999, 1 \%, tree traps; 1.XI.1999-1.V.2000, 1 क, soil traps - [E3.04] soil traps: 1.X.1998-15.V.1999, 2 o 0 ; 1.X-1.XI.1999,

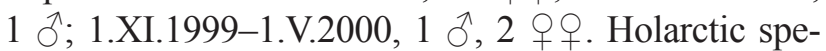
cies. Other localities in Bulgaria: Sofia Plain (Beschovski \& Langourov, 1997) and Eastern Rhodope Mts (Langourov, 2004b).

58. Triphleba tumidula (Schmitz, 1918) - Material examined: [E1.03]: 15.IV.2000, 1 ठ․ European species. Other localities in Bulgaria: Eastern Rhodope Mts (Langourov, 2004b).

** 59. Triphleba vitrea (Wood, 1906) - Material examined: [E3.03] soil traps: 5.X-1.XI.1999, 1 q. European species, known till now from Austria, Great Britain, Slovakia and Netherlands. New region: Balkan Peninsula - Bulgaria.

** 60. Triphleba withersi Disney, 1992 - Material examined: [N1.01] Malaise trap: 10-18.V.1997, 4 ठึ ठै, 


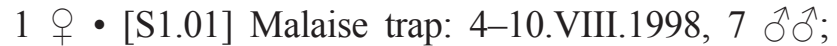

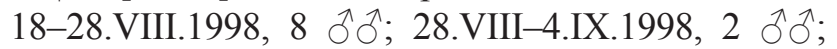

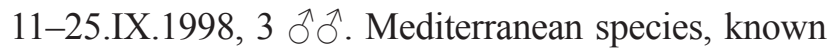
till now only from the type-locality in France. New region: Balkan Peninsula - Bulgaria.

\section{Metopininae}

** 61. Megaselia abernethae Disney, 1988 - Material examined: [N1.06], tree traps: 1.VI-1.VII.1999, 1 ภ. European species, known till now only from the typelocality in Scotland (Great Britain). New region: Balkan Peninsula - Bulgaria.

62. Megaselia aculeata (Schmitz, 1919) - Material examined: [S1.01] Malaise trap: 4-10.VIII.1998, 8

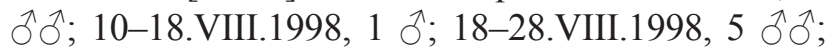
28.VIII-4.IX.1998, 1 ते $\bullet$ [E2.01], soil traps: 1.IX-1.X.1999, 1 ते $\bullet \quad[\mathrm{E} 3.01]$ soil traps: 7.VIII-6.IX.1999, 9 ठึึ, 2 우 • [N3.02]: 12.VI-4.VII.1999, 5 ठิో • [E3.02]: 1.VI-1.VII.1999, 2 ठぇ,, soil traps; 1.VII-1.VIII.1999, 2 ठぇ,, tree traps; 1.VIII-1.IX.1999, 1 ô, 3 우, tree traps; 1.IX-1.X.1999, $1 \hat{\jmath}$, tree traps; $1 \hat{\partial}$, soil traps -

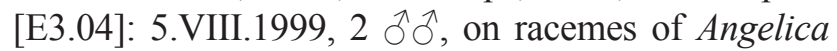
sylvestris L. • [W3.04] soil traps: 3.VII-7.VIII.1999, 4 今े ${ }^{2}, 1$ P 1 [W3.06] soil traps: 3.VII-6.VIII.1999, 2

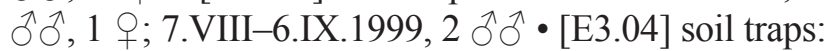
1.VII-1.VIII.1999, 1 $\widehat{\jmath}, 1$ ㅇ. Westpalaearctic species, known from Europe and Asia (Iran). Other localities in Bulgaria: Western Rhodope Mts (Langourov, 2010).

63. Megaselia aequalis (Wood, 1909) - Material examined: [E1.01]: 15.IV.2000, 1 q $\bullet$ [N1.04]: 30.V.1993, 1 त $\bullet 1260-1360 \mathrm{~m}$, near Esperanto Chalet, mixed wood: 30.V.1993, 1 đ $\cdot$ [E1.05], tree traps: 1.V1.VI.1999, 1 o $\quad$ [N1.05] Malaise trap:

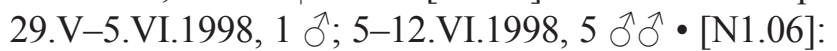

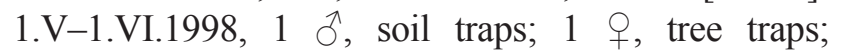
1.V-1.VI.1999, 1 o, 1 , tree traps; 1 , soil traps; 1.VI-1.VII.1999, 1 O, 2 우, soil traps $1500 \mathrm{~m}$, E of Septemvri Chalet, beech wood, tree traps: 3-10.VII.1998, 1 ㅇ •[E2.01]: 1.IX-1.X.1998, 1 ๙ึ, soil traps; 2.X.1998, 1 • [N2.03]: 20.VIII.1992, 1 ô, 1 q, in copula - [E3.02]: 1.V-1.VI.1999, 1 Oे, tree traps; 1.VII-1.VIII.1999, 1 ô, soil traps; 1.VIII-1.IX.1999, 3 $\widehat{\partial} \hat{\partial}, 2$ q $ᄋ$, tree traps; $1 \hat{\jmath}, 1$, soil traps $\cdot 2150 \mathrm{~m}$, near Malak Rezen, subalpine zone: 28.V.1998, 1 q. Holarctic; known from Europe, Asia (Israel, Russian Far East) and North America. For the Balkan Peninsula also known from Croatia, Slovenia (Schmitz, 1924, 1928e), North Macedonia (Coe, 1956; Langourov,
1999) and Montenegro (Langourov, 2004a). Other localities in Bulgaria: W Rhodope Mts (Langourov, 2010). ** 64. Megaselia affinis (Wood, 1909) - Material examined: [S1.01] Malaise trap: 28.VIII-4.IX.1998, 3

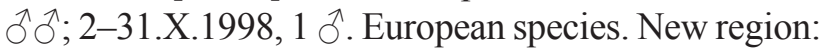
Balkan Peninsula - Bulgaria.

** 65. Megaselia albicans (Wood, 1908) - Material examined: [N1.06]: 1.XI.1998-15.IV.1999, 1 ภे,

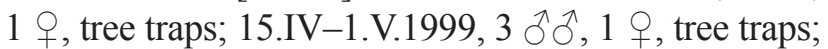
1 ô, soil traps. European species, known from Middle Europe and Sweden. New region: Balkan Peninsula Bulgaria.

66. Megaselia albicaudata (Wood, 1910) - Material examined: [S1.01] Malaise trap: 17-30.IV.1999, 1

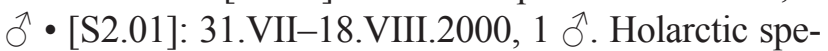
cies, known from Europe, Macaronesia (Canary Is), Asia (Israel, Iran, Yemen, China) and North America. For the Balkan Peninsula also known from Montenegro (Langourov, 2004a). Other localities in Bulgaria: Western Rhodope Mts (Langourov, 2010).

**67. Megaselia albiclava Schmitz, 1926 - Material examined: [N1.05] Malaise trap: 29.V-5.VI.1998, $1 \hat{\sigma} \cdot[\mathrm{N} 1.06]$ soil traps: 1.VII-1.VIII.1999, 1 ઈิ. European species, known till now from Middle and North Europe. New region: Balkan Peninsula - Bulgaria.

68. Megaselia albocingulata (Strobl, 1906) - Material examined: [S1.01] Malaise trap: 18-28.VIII.1998, 2 ठิ ठึ; 978 m, near Bosnek Village, near Pepelyankata Cave: 2.X-2.XI.2013, 1 ภ̂, 4 우우 1006 m, near Bosnek Village, near Duhlata Cave, MSS traps: 6.VI-2.X.2013, 1 § , 4 우. Mediterranean species; also known from Iran. For the Balkan Peninsula known from Croatia (Schmitz, 1924 as Aphiochaeta languescens (Schmitz, 1924)) and Greece (Disney, 1991). Published for the mountain by Langourov et al. (2014).

** 69. Megaselia alticolella (Wood, 1909) - Material examined: [W3.05] soil traps: 7.VIII-6.IX.1999, 1 $\widehat{~}$. European species. New region: Balkan Peninsula - Bulgaria.

70. Megaselia altifrons (Wood, 1909) - Material examined: [S1.01] Malaise trap: 4-10.VIII.1998, 1 §ै; 28.VIII-4.IX.1998, 1 ○ $\bullet$ [E2.01], soil traps: 1.VIII-1.IX.1998, 1 ठิ. Westpalaearctic species, known from Europe and Asia (Iran). For the Balkan Peninsula is also known from Slovenia (Schmitz, 1928). Other localities in Bulgaria: Sofia Plain (Beschovski \& Langourov, 1997; Langourov, 2004b) and Western Rhodope Mts (Langourov, 2010). 
71. Megaselia angusta (Wood, 1909) - Material examined: [E1.02]: 15.IV.2000, 1 $\hat{O}$, on flowers of Petasites hybridus ochroleucus (Boiss. et Huet) Sourek; 954 m, near Bosnek Village, near Academik Cave, MSS traps: 6.VI-2.XI.2013, 1 §; $1120 \mathrm{~m}$, above Bosnek, on the road to Chuipetlyovo, scree in mixed wood, MSS traps: 2.X-2.XI.2013, 2 $\widehat{\jmath}$. Westpalaearctic species, known from Europe, Macaronesia and Asia (Israel). For the Balkan Peninsula is known also from Slovenia (Schmitz, 1928), Croatia (Coe, 1956) and Montenegro (Langourov, 2004a). In Bulgaria also known from W Rhodope Mts (Langourov, 2010) and other parts of Vitosha Mt (Langourov et al., 2014).

** 72. Megaselia aquilonia Schmitz, 1958 - Material examined: [E3.02], soil traps: 1.V-1.VI.1999, 1 ơ; 1.VI-1.VII.1999, 1 Oे. European species, known till now from Great Britain, Poland, Norway and Sweden. New region: Balkan Peninsula - Bulgaria.

73. Megaselia basispinata (Lundbeck, 1920) Material examined: [S2.01]: 31.VII-18.VIII.2000, 1 ठ - [N1.06]: 1.VIII-1.IX.1998, 1 Oे, tree traps; 1.VI-1.VII.1999, 1 ठ, soil traps - [E3.02], tree traps: 1.VII-1.VIII.1998, 1 §, 1 q. Holarctic-Neotropical species. For the Balkan Peninsula is also known from Slovenia (Schmitz, 1928). Other localities in Bulgaria: Eastern Rhodope Mts (Langourov, 2004b) and Sofia Plain (Langourov, 2005).

** 74. Megaselia beckeri (Wood, 1909) - Material examined: [N1.06]: 27.VIII.1992, 1 $\hat{0}$ - between [E2.01] to [E2.02]: 3.IX.1992, 1 ô. Holarctic species, known from Middle and North Europe and North America. New region: Balkan Peninsula - Bulgaria.

75. Megaselia berndseni (Schmitz, 1919) - Material examined: [E1.05], tree traps: 1.VII-1.VIII.1999, $1 \hat{\sigma} \cdot[\mathrm{N} 1.06]$ soil traps: 1.VIII-1.IX.1998, 1 क; 1.V-1.VI.1999, 1 ते $\bullet$ [E2.01], soil traps: 1.VII-1.VIII.1998, 1 ๙ $1830 \mathrm{~m}$, Aleko Chalet, on window (leg. S. Lazarov): 2.V.1999, 1 ठ • [E3.02], tree traps: 1.VIII-1.IX.1999, 1 q. Holarctic species, known for the Balkan Peninsula from Greece (Schmitz, 1924) and North Macedonia [Coe, 1956 as Megaselia pygmaeoides (Lundbeck)]. Other localities in Bulgaria: Eastern Rhodope Mts (Langourov, 2004b).

** 76. Megaselia bovista (Gimmerthal, 1848) Material examined: [E3.02], tree traps: 1.VI-1.VII.1999, 1 §. Westpalaearctic species, known from Europe and Asia (Israel and Iran). New region: Balkan Peninsula - Bulgaria.

77. Megaselia brevicostalis (Wood, 1910) - Material examined: [E2.01], soil traps: 1.IX-1.X.1999, 1 ๙
[E3.02], soil traps: 1.V-1.VI.1999, 1 o $\bullet$ [E3.04] soil traps: 1.VII-1.VIII.1999, 1 §. Holarctic-Neotropical species. For the Balkan Peninsula also known from Slovenia (Schmitz, 1928), North Macedonia (Coe, 1956) and Montenegro (Langourov, 2004a). Other localities in Bulgaria: Western Stara Planina Mts (Schmitz, 1953), Eastern Rhodope Mountains (Langourov, 2004b) and Western Rhodope Mts (Langourov, 2010).

78. Megaselia brevior (Schmitz, 1924) - Material examined: [S1.01] Malaise trap: 28.VIII-4.IX.1998, 1

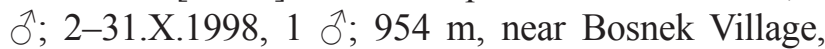
near Academik Cave, MSS traps: 6.VI-2.XI.2013, 1 9 . Westpalaearctic; known from Europe, Macaronesia (Azores) and Asia (Iran). For the Balkan Peninsula also known from North Macedonia (Langourov, 1999). Published for the mountain by Langourov et al. (2014).

79. Megaselia breviterga (Lundbeck, 1920) - Material examined: $900 \mathrm{~m}$, near Bosnek Village, near Popov Izvor Karst spring, MSS traps: 6.VI-2.X.2013, 18 रे $\partial^{\lambda}, 4$ 웅 1118 m, near Bosnek Village, Zhivata

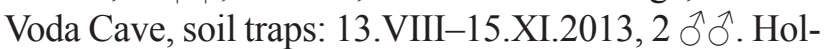
arctic species. For the Balkan Peninsula also known from Croatia (Schmitz, 1924). Other localities in Bulgaria: Western Rhodope Mts (Langourov, 2010).

** 80. Megaselia brunneipennis Costa, 1857 - Material examined: [S1.01] Malaise trap:

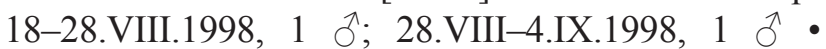
[E1.05], tree traps: 1.IX-1.X.1998, 1 ○ $\cdot[\mathrm{N} 1.06]$ soil traps: 1.VIII-1.IX.1999, 1 §. Westpalaearctic species, known from Europe and Macaronesia (Canary Is). New region: Balkan Peninsula - Bulgaria.

** 81. Megaselia campestris (Wood, 1908) - Material examined: [S1.01] Malaise trap: 2-31.X.1998, 1 $\hat{\sigma} \cdot[\mathrm{E} 1.05]$, tree traps: 1.VII-1.VIII.1998, $1 \hat{\sigma} \cdot$ [N1.06] soil traps: 1.VII-1.VIII.1999, 1 q. European species. New region: Balkan Peninsula - Bulgaria.

82. Megaselia ciliata (Zetterstedt, 1848) - Material examined: [N1.01] Malaise trap: 10-18.V.1997, 1 § $\bullet$ [E1.05]: 1.VIII-1.IX.1998, 1 §ิ, 1 q, soil traps;

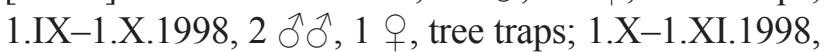

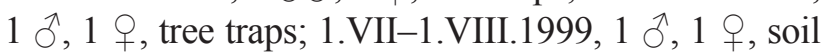

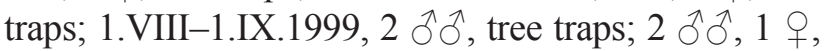
soil traps; 1.IX-1.X.1999, $1 \hat{\partial}$, tree traps; $2 \hat{\partial} \widehat{\partial}$, soil traps; 1.X-1.XI.1999, 2 우, tree traps $-[\mathrm{N} 1.05]$ Malaise trap: 5-12.VI.1998, 1 o $\bullet$ [N1.06]: 1.VII-1.VIII.1998, 1 \%, soil traps; 1.VIII-1.IX.1998, 1 Oे, tree traps; 6.X.1998, 1 क; 1.VIII-1.IX.1999, 2

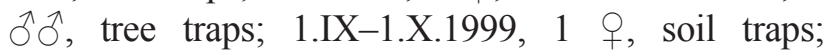
1.X-1.XI.1999, 1 q, tree traps - [E2.01]: 
1.V-1.VI.1998, 2 §ิర, 2 우, tree traps; 1.VI-1.VII.1998, 1 ô, soil traps; 2.X.1998, 1 क; 1.VIII-1.IX.1999, 2 우, tree traps; 1.IX-1.X.1999, 1 ô, 1 + , tree traps - [E3.02]: 1.IX-1.X.1998, 1 q, soil traps; 1.V-1.VII.1999, 1 क , tree traps; 1 Oे, soil traps; 1.VI-1.VII.1999, 1 ô, soil traps; 1.VIII-1.IX.1999,

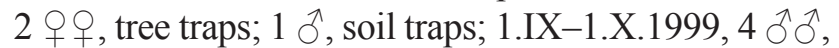
5 우오, tree traps; $3 \hat{\partial} \widehat{\partial}, 3$ 우, soil traps $\bullet$ [W3.04] soil traps: 6.IX-4.X.1999, 1 ठ $\cdot$ [E3.04] soil traps: 1.IX-1.X.1999, 1 9. European species, reported for the Balkan Peninsula from Bosnia-Herzegovina (Strobl, 1898) and Montenegro (Langourov, 2004a). Other localities in Bulgaria: Sofia Plain (Langourov, 2004c).

** 83. Megaselia coei Schmitz, 1938 - Material examined: [N1.06], tree traps: 1.IX-1.X.1998, 1 रे [E3.04]: 5.VIII.1999, 2 $\widehat{\partial}, 1$, , on racemes of Angelica sylvestris L. European species, known from Austria, Great Britain and Poland. New region: Balkan Peninsula - Bulgaria.

** 84. Megaselia conformis (Wood, 1909) - Material examined: [E3.02], tree traps: 1.VIII-1.IX.1998, 1 ô, 1 q. European species. New region: Balkan Peninsula-Bulgaria.

* 85. Megaselia crassicosta (Strobl, 1892) - Material examined: [N1.03]: 30.V.1993, 1 • $[$ [E1.05] soil traps: 1.IX-1.X.1998, 1 ○े; 1.V-1.VI.1999, 1 \%; 1.VII1.VIII.1999, 1 o $\bullet$ [E2.01], tree traps: 1.VI-1.VII.1999, 1 ㅇ [N3.02]: 12.VI-4.VII.1999, 1 o $•$ [W3.02] soil traps: 5.VI-3.VII.1999, 1 ค • [E3.04] soil traps: 1.IV-1.VII.1999, 1 ○; 1.IV-1.VIII.1999, 1 q. European species, reported for the Balkan Peninsula from Croatia (Schmitz, 1924e) and Montenegro (Langourov, 2004a).

* 86. Megaselia crassipes (Wood, 1909) - Material examined: [E1.05]: 1.XI.1998-15.IV.1999, 2 $\widehat{\partial}$, tree traps; 1.VII-1.VIII.1999, 1 ภ, soil traps. Holarctic; known from Europe and North America. For the Balkans known from Montenegro (Langourov, 2004a).

87. Megaselia curvicapilla Schmitz, 1947 - Material examined: [S1.01] Malaise trap: 18-28.VIII.1998, 1

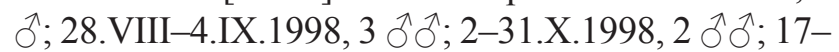
30.IV.1999, 1 ๙ $\bullet$ [N1.06] soil traps: 1.V-1.VI.1999, 1 ก • [E3.03] soil traps: 5.VII-6.VIII.1999, 1 ๙ $\bullet$ [E3.04] soil traps: 1.IV-1.IX.1999, 1 ठ. Westpalaearctic species, known from Europe and Asia (Israel, Iran). For the Balkan Peninsula also known from North Macedonia (Langourov, 1999). Other localities in Bulgaria: Western Rhodope Mts (Langourov, 2010).

88. Megaselia dahli (Becker, 1901) - Material ex-

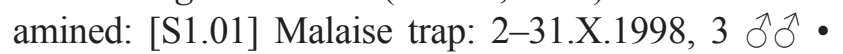

[N1.06], tree traps: 1.VI-1.VII.1999, 1 ô. European species, known for the Balkan Peninsula from North Macedonia (Langourov, 1999) and Montenegro (Langourov, 2004a). Other localities in Bulgaria: Western Rhodope Mts (Langourov, 2010).

* 89. Megaselia differens Schmitz, 1948 - Material examined: [E3.02], soil traps: 1.V-1.VII.1999, 1 ô. Westpalaearctic species, known from Europe and Asia (Russian Far East). For the Balkan Peninsula also known from North Macedonia (Langourov, 1999).

* 90. Megaselia discreta (Wood, 1909) - Material examined: [E1.05]: 1.VIII-1.XI.1999, 1 Oे, 2 우, tree traps; $1 \hat{\partial}$, soil traps; 1.IX-1.X.1999, $1 \hat{\partial}, 1$ ㅇ, soil traps. European species, known for the Balkan Peninsula from Croatia (Schmitz, 1924).

91. Megaselia diversa (Wood, 1909) - Material examined: [E1.04]: 20.VIII.1992, 1 q • [E1.05]:

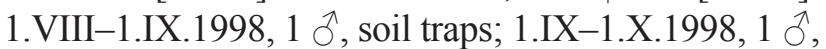
soil traps; 1.V-1.VI.1999, 1 q, tree traps; 1.VII-1.VIII.1999, 1 §, tree traps; 1.VIII-1.IX.1999, $2 \widehat{\jmath} \partial^{\lambda}, 3$ 우 , tree traps; 1.IX-1.X.1999, $1 \hat{\jmath}$, tree traps - [N1.06]: 1.VIII-1.IX.1998, 1 o, soil traps; 1.X-1.XI.1998, 2 ふふ, 1 ค , tree traps; 15.IV-1.V.1999, 1 o, tree traps; 1.V-1.VI.1999, 1 o, soil traps;

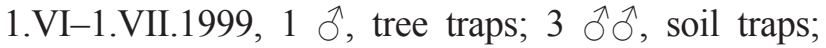
1.VIII-1.IX.1999, 1 ô, soil traps $\bullet$ between [N1.06] to [N1.07]: 19.IV.1993, 1 ठ • [E2.01]: 1.VII-1.VIII.1998,

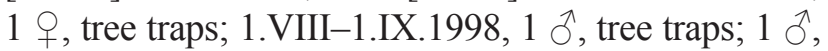

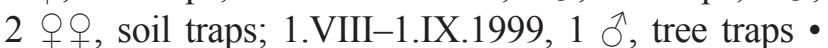
between [E2.01] to [E2.02]: 17.X.1992, 1 - [N2.02]: 27.VIII.1992, 1 ते $\cdot$ [N2.03]: 31.V.1998, 1 ते 1820-1870 m, between Edelvais Chalet to Konyarnika place, spruce wood: 2.VI.1996, 1 ठ $\cdot$ [W3.01]: 2.VI.1996, 1 o $\cdot[$ E3.02]: 1.VII-1.VIII.1998, 1 ô, tree traps; 1.VIII-1.IX.1998, 1 क, tree traps; 1.IX-1.X.1998, 1 ○, 3 우, tree traps; 1.V-1.VI.1999,

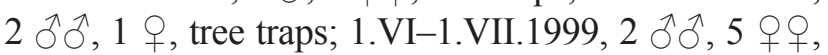
tree traps; 1.VII-1.VIII.1999, 1 o, 1 क, soil traps; 1.VIII-1.IX.1999, 1 §, tree traps; 1 O, 1 \%, soil traps;

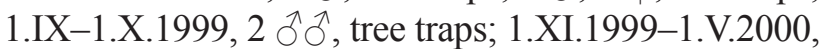
$1 \hat{\jmath}$, soil traps $\bullet$ [E3.03] soil traps: 5.VII-6.VIII.1999, 1

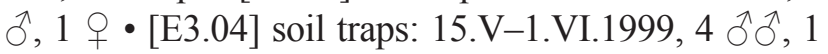
+; 1.VI-1.VII.1999, 1 oे $\bullet$ [S4.02] soil traps: 7.VIII-6.IX.1999, $1 \hat{\delta} \cdot 2280 \mathrm{~m}$, Cherni Vruh, subalpine zone: 2.VI.1996, 1 ठ. Holarctic; known from Europe, North Africa (Tunisia), Asia (Israel, Russian Far East) and North America. For the Balkan Peninsula known from Croatia (Schmitz, 1924; Coe, 1956), other parts of the former Yugoslavia (Schmitz \& Beyer, 1965), Montenegro (Langourov, 2004a). Other localit- 
ies in Bulgaria: Kresna Gorge (Langourov \& Sakalian, 2001), E Rhodope Mts (Langourov, 2004b), W Rhodope Mts (Langourov, 2010).

** 92. Megaselia dubitalis (Wood, 1908) - Material examined: [N1.05] Malaise trap: 5-12.VI.1998,

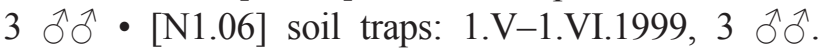
European species, known from Middle and North Europe. New region: Balkan Peninsula - Bulgaria.

** 93. Megaselia eccoptomera Schmitz, 1927 Material examined: [E2.01], tree traps: 1.VII-1.VIII.1999, 1 ภ • [W3.01]: 2.VI.1996, 1 ภ [E3.02], soil traps: 1.V-1.VI.1999, 1 ऊ; 1.VI-1.VII.1999, $6 \widehat{\partial} \widehat{\partial}, 1$ q. Holarctic species, known from Europe and North America. New region: Balkan Peninsula - Bulgaria.

* 94. Megaselia elongata (Wood, 1914) - Material examined: [N1.05] Malaise trap: 5-12.VI.1998, 1 §.. Westpalaearctic; known from Europe and Asia (Israel). Balkan Peninsula: Croatia (Schmitz, 1924 as Aphiochaeta cuspidata Schmitz, 1919).

95. Megaselia emarginata (Wood, 1908) - Material examined: [N1.01] Malaise trap: 10-18.V.1997, 1 ते - [S2.01]: 16-23.V.2000, 5 ठิ • [N1.05] Malaise trap: 29.V-5.VI.1998, 1 ठे; 5-12.VI.1998, 1 गे; 3-8.VII.1998, 1 ठ․ European species, for the Balkan Peninsula known from Croatia (Coe, 1956) and Montenegro (Langourov, 2004a). Other localities in Bulgaria: Sofia Plain (Langourov, 2004b).

** 96. Megaselia errata (Wood, 1912) - Material examined: [N1.01] Malaise trap: 10-18.V.1997, 1 ๙ $\bullet$ [E1.05] soil traps: 1.VIII-1.IX.1998, $1 \hat{\jmath}, 1$ q. European species, known from Middle Europe. New region: Balkan Peninsula - Bulgaria.

97. Megaselia flava (Fallen, 1823) - Material examined: [E1.05]: 28.VII.1993, 1 ơ; 1.VIII-1.IX.1998, $1 \hat{\jmath}$, tree traps; 1.IX-1.X.1998, 1 ठै, soil traps • 1260-1360 m, near Esperanto Chalet, mixed wood: 3.IX.1992, $1 \hat{0} \cdot$ between [E1.05] to [E1.06]: 27.VIII.1992, 1 ก $\bullet \quad[\mathrm{N} 1.05]$ Malaise trap: 3-8.VII.1998, 1 ते • [N1.06] soil traps: 1.VII-1.VIII.1999, 3 우. Holarctic-Oriental species, known from Europe, Asia (Israel, Japan), North America and Oriental Region. For the Balkan Peninsula known from Bosnia-Herzegovina (Strobl, 1898), Croatia (Coe, 1956), North Macedonia (Coe, 1956; Langourov, 1999) and Montenegro (Langourov, 2004a). Other localities in Bulgaria: Western Rhodope Mts (Langourov, 2010).

98. Megaselia flavicans Schmitz, 1935 - Material

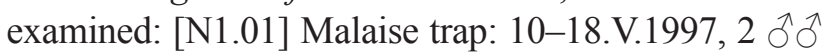

• [S1.01] Malaise trap: 17-30.IV.1999, 2 ふぇ • [E1.05], tree traps: 1.VIII-1.IX.1998, 1 - [N1.06] soil traps:

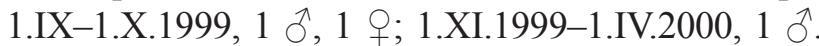
Westpalaearctic; known from Europe and Asia (Turkey. Balkan Peninsula: Croatia (Schmitz, 1953), North Macedonia (Coe, 1956), Greece (Disney, 1991). Bulgaria: Kresna Gorge (Langourov \& Sakalian, 2001).

** 99. Megaselia flavicoxa (Zetterstedt, 1848) Material examined: [E1.05] soil traps: 1.VIII-1.IX.1999, 1 §ิ. European species. New region: Balkan Peninsula - Bulgaria.

** 100. Megaselia frameata Schmitz, 1927 - Material examined: [E1.06]: 20.VIII.1992, 1 §. European species. New region: Balkan Peninsula - Bulgaria.

** 101. Megaselia frontalis (Wood, 1909) - Material examined: [S1.01] Malaise trap: 18-28.VIII.1998, $1 \hat{\jmath}$. European species, known from Middle Europe. New region: Balkan Peninsula - Bulgaria

** 102. Megaselia fungivora (Wood, 1909) - Material examined: [E1.05], tree traps: 1.VII-1.VIII.1999, $1 \hat{\jmath}$. Holarctic species, known from Middle and North Europe and North America. New region: Balkan Peninsula - Bulgaria.

103. Megaselia fusca (Wood, 1909) - Material examined: [E1.05], tree traps: 1.IX-1.X.1998, 1 సै; 1.VII-1.VIII.1999, 1 ठ, soil traps; • [N1.06] soil traps: 1.IX-1.X.1999, 2 ठิठิ, 1 ค • [E3.02], soil traps: 1.VI-1.VII.1999, 1 गें 1.VII-1.VIII.1999, 1 ते • [W3.03] soil traps: 12.VI-3.VII.1999, 1 o $\bullet$ [E3.04] soil traps: 1.VI-1.VII.1999, 5 ふぇં; 1.VII-1.VIII.1999, 1 ${ }^{7}$. Palaearctic; known from Europe and Asia (Russian Far East). For the Balkan Peninsula also known from Bosnia-Herzegovina (Schmitz, 1928), Croatia (Coe, 1956) and Montenegro (Langourov, 2004a). Other localities in Bulgaria: Western Stara Planina Mts (Czerny, 1930) and Vitosha Mt (Langourov, 2001).

** 104. Megaselia fuscipalpis (Lundbeck, 1920) Material examined: [N1.06] soil traps: 1.V-1.VI.1999, 1 ठ . European species, known from Middle and North Europe. New region: Balkan Peninsula - Bulgaria.

** 105. Megaselia fuscovariana Schmitz, 1933 Material examined: [N1.01] Malaise trap: 10-18.V.1997, 1 ठิ. European species, known from Middle Europe. New region: Balkan Peninsula - Bulgaria.

106. Megaselia giraudii (Egger, 1862) - Material

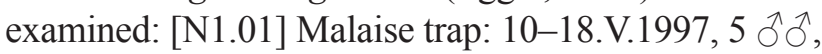
1 ㅇ [S1.01] Malaise trap: 18-28.VIII.1998, 1 गे;

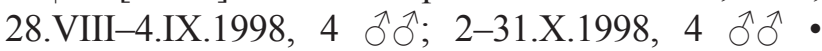
[E1.05]: 1.VII-1.VIII.1998, 1 ô, tree traps; 
1.VIII-1.IX.1998, 1 ô, 1 क, soil traps;

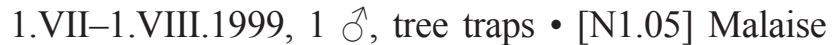
trap: 3-8.VII.1998, 1 ठ • [N1.06]: 1.V-1.VI.1999, 1 ふ,, soil traps; 1.VII-1.VIII.1999, 2 ठิึ, tree traps • [E2.01]: 1.VIII-1.IX.1998, 1 ○, 1 , soil traps; 1.VIII-

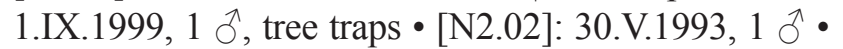
[E3.02], tree traps: 1.VII-1.VIII.1999, 1 ô. Holarctic species, known from Europe, Macaronesia, Asia (Israel, Iran, Russian Far East) and North America. For the Balkan Peninsula also known from BosniaHerzegovina (Strobl, 1898), Croatia, Serbia (Schmitz, 1924) and North Macedonia (Coe, 1956). Other localities in Bulgaria: Sofia Plain (Langourov, 2004b) and Western Rhodope Mts (Langourov, 2010).

** 107. Megaselia glabrifrons (Wood, 1909)-Material examined: [N1.05] Malaise trap: 5-12.VI.1998, $2 \hat{\jmath} \widehat{\partial} \cdot[\mathrm{N} 1.06]$ soil traps: 1.VII-1.VIII.1998, 1 ते;

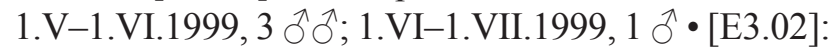
1.VI-1.VII.1999, $1 \hat{\sigma}$, tree traps; $10 \widehat{\partial} \widehat{\partial}$, soil traps; 1.VII-1.VIII.1999, 1 ठ , tree traps. Holarctic species, known from Europe and North America. New region: Balkan Peninsula - Bulgaria.

** 108. Megaselia gregaria (Wood, 1910) - Material examined: [S1.01] Malaise trap: 2-31.X.1998, 1 §. European species. New region: Balkan Peninsula Bulgaria.

109. Megaselia halterata (Wood, 1910) - Material examined: [S1.01] Malaise trap: 18-28.VIII.1998, 1 ○े; 28.VIII-4.IX.1998, 1 ठ. Subcosmopolitan species, known from Europe, Macaronesia, North Africa, Asia and North America, introduced to Australia and New Zealand. Other localities in Bulgaria: Western Stara Planina Mts, Sofia Plain and Vitosha Mt (Langourov, 2001).

** 110. Megaselia hirsuta (Wood, 1910) - Material examined: [S1.01] Malaise trap: 18-28.VIII.1998, 1 ठ - [E1.05] soil traps: 1.VII-1.VIII.1999, 1 ○ $[$ [N1.06] soil traps: 1.VII-1.VIII.1998, $2 \hat{\jmath}, 1 q \cdot$ [E2.01], tree traps: 1.VII-1.VIII.1998, 1 ๙ $\cdot$ [E3.02], soil traps: 1.V-1.VI.1999, 1 §, 1 ㅇ. Westpalaearctic species, known from Europe and Macaronesia. New region: Balkan Peninsula - Bulgaria.

111. Megaselia hirticaudata (Wood, 1910) - Material examined: [N1.06] soil traps: 15.IV-1.V.1999, 1 $\delta \cdot[E 2.01]$, tree traps: 1.VIII-1.IX.1999, $1 \hat{\delta}$. Westpalaearctic species, known from Europe and Asia (Iran). For the Balkan Peninsula also known from Montenegro (Langourov, 2004a as M. capronata Schmitz, 1940). Other localities in Bulgaria: Eastern Rhodope Mts (Langourov, 2004c).
112. Megaselia hirtiventris (Wood, 1909) - Material examined: [E1.05] soil traps: 1.X-1.XI.1998, 1 శึ. Palaearctic species, known from Europe and Asia (Russian Far East). Other localities in Bulgaria: Sofia Plain (Langourov, 2004b) and Eastern Rhodope Mts (Langourov, 2004c).

** 113. Megaselia horsfieldi Disney, 1986 - Material examined: [E3.02], tree traps: 1.VIII-1.IX.1999, 1 ऽ. European species, till now known only from Great Britain. New region: Balkan Peninsula - Bulgaria.

** 114. Megaselia hyalipennis (Wood, 1912) Material examined: [E2.01], tree traps:

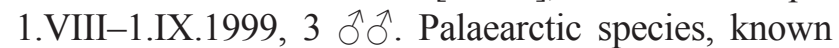
from Middle and North Europe and Asia (Russian Far East). New region: Balkan Peninsula - Bulgaria.

** 115. Megaselia hypopygialis (Lundbeck, 1920)

- Material examined: [E1.05] soil traps:

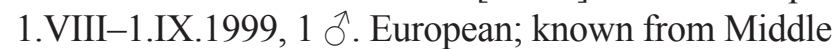
Europe. New region: Balkan Peninsula - Bulgaria.

** 116. Megaselia intonsa Schmitz, 1948 - Material examined: [E1.05]: 1.VII-1.VIII.1998, 1 ㅇ, tree traps; 1.V-1.VI.1999, 2 우, soil traps $-[\mathrm{N} 1.06]$ soil traps: 1.V-1.VI.1999, 1 ठิ $\bullet$ [E1.06]: 20.VIII.1992, 1 ภ - $1500 \mathrm{~m}, \mathrm{E}$ of Septemvri Chalet, beech wood, tree traps: 3-10.VII.1998, 2 ठิ 0 • [E2.01]: 20.VIII.1992,

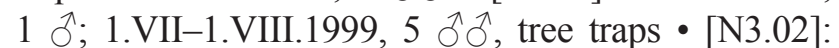
4.VII-6.VIII.1999, 7 ふึ, 6 o 우 • [E3.02]:

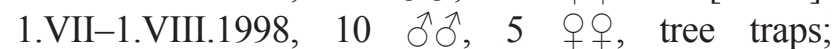
1.VIII-1.IX.1998, 1 ठ , tree traps; 1.VI-1.VII.1999,

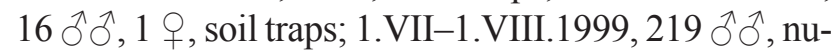
merous 9 우, tree traps; $34 \hat{\delta} \widehat{O}, 32$ 우, soil traps • [N3.03] soil traps: 4.VII-6.VIII.1999, 1 ô, 4 우 •

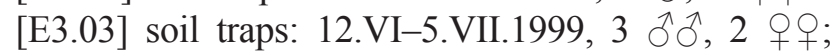
5.VII-6.VIII.1999, 18 ๙ึ, 7 우 • [W3.02] soil traps:

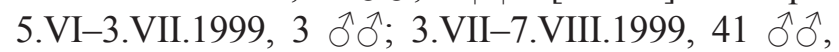
16 우 • [W3.03] soil traps: 3.VII-7.VIII.1999, 19 $\hat{\jmath}$, 13 웅 7.VIII-6.IX.1999, 1 ○ $\bullet$ [W3.04] soil traps:

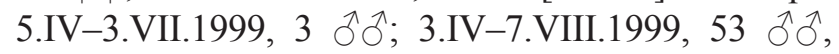
12 우 • $[$ [W3.05] soil traps: 3.VII-7.VIII.1999, 15 ふో ठิ, 4 우우 • [E3.04] soil traps: 1.VII-1.VIII.1999, 30 ô ô, 4 oㅇ; 1.VIII-1.IX.1999, 2 ठึग, 1 क;

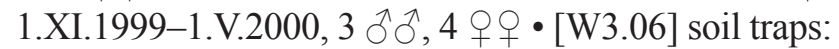

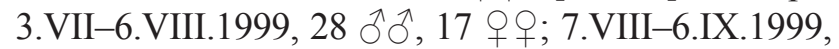

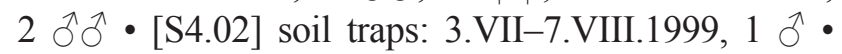
[S4.03]: 5.VII-6.VIII.1999, 9 ふふ, 2 우. Palaearctic species, known from North Europe and Asia (Russian Far East). New region: Balkan Peninsula - Bulgaria.

117. Megaselia involuta (Wood, 1910) - Material examined: [E2.01], soil traps: 1.VII-1.VIII.1999, 1 §. European species, known for the Balkan Peninsula 
from Greece and Serbia (Schmitz, 1924). Other localities in Bulgaria: Kresna Gorge (Langourov \& Sakalian, 2001 ) and Western Rhodope Mts (Langourov, 2010).

118. Megaselia largifrontalis Schmitz, 1939 - Material examined: $900 \mathrm{~m}$, near Bosnek Village, near Popov Izvor Karst spring, MSS traps: 6.VI-2.X.2013, $2 \widehat{\widehat{\partial}}, 1$ ㅇ. Palaearctic-Afrotropical species, known from Europe, Asia (Yemen) and Afrotropical Region (St Helena Island, introduced). Published for the mountain by Langourov et al. (2014).

119. Megaselia lata (Wood, 1910) - Material examined: [S2.01]: 18.VIII-1.IX.2000, $1 \hat{\jmath} \cdot$ [E1.05]:

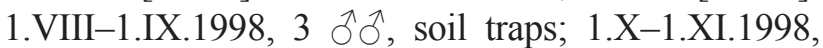

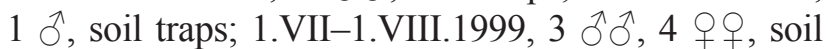
traps; 1.VIII-1.IX.1999, 1 गे, soil traps;

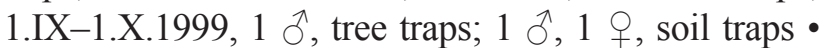
[N1.06]: 1.VII-1.VIII.1998, 8 ठิ่, 12 q , , soil traps; 1.IX-1.X.1998, 1 ठै, tree traps; 1.V-1.VI.1999, 1 ô, soil traps; 1.VI-1.VII.1999, 1 Ô, soil traps; 1.VII-1.VIII.1999, 1 ภิ, 3 9 , , soil traps; 1.VIII-1.IX.1999, 1 गे, tree traps - [E2.01]:

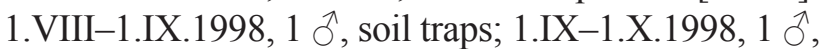
soil traps; 1.VIII-1.IX.1999, $2 \hat{\jmath} \widehat{\partial}$, tree traps; $1 \hat{\partial}$, soil traps • [E3.02]: 1.VII-1.VIII.1999, 1 ठे, tree traps; 1 ठ, soil traps • [W3.05] soil traps: 7.VIII-6.IX.1999, 1 సิ. Westpalaearctic species, known from Europe and Macaronesia. For the Balkan Peninsula also known from Slovenia (Schmitz, 1928) and Montenegro (Langourov, 2004a). Other localities in Bulgaria: Western Rhodope Mts (Langourov, 2010).

* 120. Megaselia latifemorata (Becker, 1901) Material examined: [N1.01] Malaise trap: 10-18.V.1997, 1 ふै; 28.IV-1.V.1998, 1 ठै, 1 O; 12-17.V.1998, 3 के

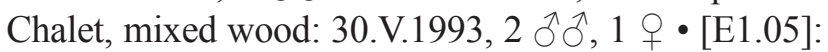
1.VIII-1.IX.1998, 1 क , tree traps; 1.VII-1.VIII.1999, 1 O, soil traps; 1.V-1.VI.1999, 1 q, soil traps; 1.VII-1.VIII.1999, 1 ${ }^{\lambda}$, tree traps $\bullet$ [N1.05] Malaise trap: 29.V-5.VI.1998, 1 ठ; 5-12.VI.1998, 1 ठ • [N1.06]: 30.V.1993, 1 วิ, 1 क; 1.VII-1.VIII.1998, 1 ठે, soil traps; 15.IV-1.V.1999, 1 o, tree traps;

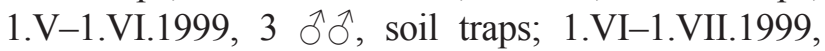
$1 \hat{O}$, soil traps - between [N1.06] to [N1.07]: 30.V.1993, 1 ô, 2 우우 $\bullet$ between [E1.06] to [E2.01]: 30.V.1993, 1 \& $\bullet$ between [N1.07] to [N2.01]: 30.V.1993, 1 đ $1500 \mathrm{~m}$, E of Septemvri Chalet, beech wood, tree traps: 3-10.VII.1998, 1 $\bullet$ [E2.01]: 1.VIII1.IX.1998, 1 ठे, soil traps; 1.V-1.VI.1999, 2 $\widehat{\partial}, 1$ q, tree traps - between [N2.02] to [N2.03]: 30.V.1993,

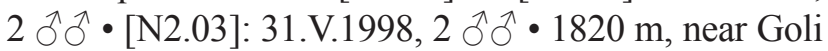

Vruh, subalpine zone (leg. N. Simov): 27.V.2000, 1 •

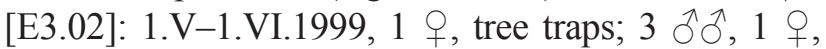
soil traps; 1.VI-1.VII.1999, 1 क, tree traps; 1.VII-1.VIII.1999, 2 Љึ, 2 qᄋ, soil traps; 1.VIII-1.IX.1999, 1 \% , tree traps - [W3.02] soil traps: 5.VI-3.VII.1999, 1 ठ $\bullet$ [W3.04] soil traps: 5.IV-3.VII.1999, 1 ㅇ $\quad$ [E3.04] soil traps:

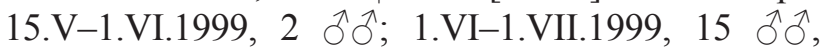

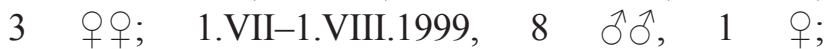
1.XI.1999-1.V.2000, 1 ภ, 1 ㅇ. Palaearctic species, known from Europe and Asia (Japan). For the Balkan Peninsula known from Croatia and Slovenia (Schmitz, 1924), North Macedonia (Langourov, 1999) and Montenegro (Langourov, 2004a).

121. Megaselia latifrons (Wood, 1910) - Material

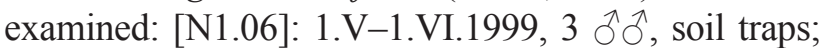
1.VI-1.VII.1999, 1 o, tree traps • [N3.01]: 3.IX.1992,

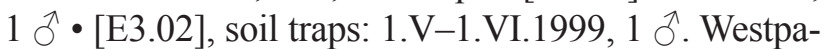
laearctic species, known from Europe and Asia (Israel). For the Balkan Peninsula known from North Macedonia (Coe, 1956; Colyer, 1956 under the synonym Megaselia propior Colyer, 1956) and Montenegro (Langourov, 2004a). In Bulgaria: Rila Mts (Schmitz, 1953) and W Rhodope Mts (Langourov, 2010).

122. Megaselia ledburiensis (Brues, 1915) - Material examined: [S1.01] Malaise trap: 18-28.VIII.1998, 3 ठぇ. Westpalaearctic species, known from Europe and Asia (Israel, Iran). Other localities in Bulgaria: Western Rhodope Mts (Langourov, 2010 as Megaselia subfuscipes Schmitz, 1935).

* 123. Megaselia longiseta (Wood, 1909) - Material examined: [N2.01]: 21.VII.1992, 1 §. Holarctic species, known from Europe, Macaronesia, Asia (Iran, Russian Far East) and North America. For the Balkan Peninsula known from Slovenia (Schmitz, 1928) and Montenegro (Langourov, 2004a).

124. Megaselia lucifrons (Schmitz, 1918) - Material examined: [N1.06] soil traps: 1.VII-1.VIII.1998,

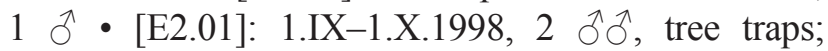
1.VIII-1.IX.1999, 1 ठ, soil traps. European species. Other localities in Bulgaria: Western Rhodope Mts (Langourov, 2010).

125. Megaselia lutea (Meigen, 1830) - Material examined: [E1.04]: 20.VIII.1992, 1 ○ [N1.05] Malaise trap: 26.VI-3.VII.1998, 1 • between [E2.01] to [E2.03]: 20.VIII.1992, 1 O. Holarctic species, for the Balkan Peninsula known from Croatia (Langhoffer, 1919), North Macedonia (Coe, 1956) and Montenegro (Langourov, 2004a). Other localities in Bulgaria: Western Rhodope Mts (Langourov, 2010). 
126. Megaselia mallochi (Wood, 1909) - Material examined: [E1.05], tree traps: 1.VII-1.VIII.1998, 1 ð;

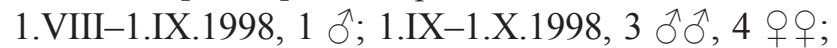

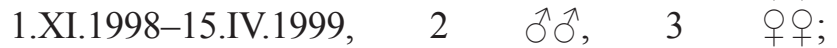
1.VII-1.VIII.1999, 1 ठึ; 1.IX-1.X.1999, 1 ภ̂, 5 우우; 1.XI.1999-1.IV.2000, 2 ठぇ • [N1.06]: 1.V-1.VI.1998, $1 \hat{\jmath}, 5$ o 9 , soil traps; 1 क, tree traps;

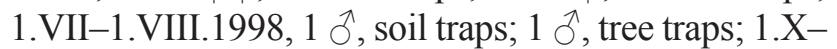
1.XI.1998, 1 ô, tree traps; 1.XI.1998-15.IV.1999,

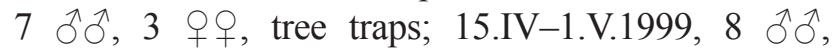

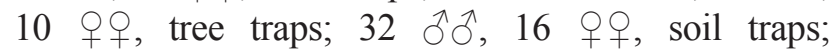
1.V-1.VI.1999, 3 ठิ 1.VIII-1.IX.1998, 1 9, tree traps; 1.IX-1.X.1998, 1 9, tree traps; 1.VII-1.VIII.1999, 4 ठิठ 1.VIII-1.IX.1999, 1 ภ, tree traps; 1 Oे, 1 क , soil traps; 1.IX-1.X.1999, 1 ठิ, tree traps; 1.X-1.XI.1999, 1 ठิ,

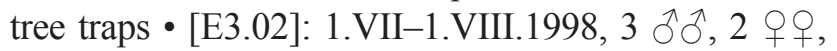
tree traps; 1.VII-1.VIII.1999, $1 \hat{\partial}$, soil traps;

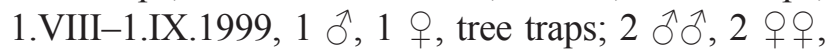
soil traps; 1.IX-1.X.1999, 1 Oे, soil traps • [E3.04] soil

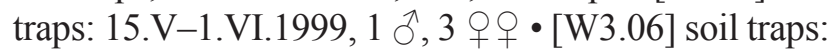
6.VI-3.VII.1999, 1 oे. European; other localities in Bulgaria: Western Rhodope Mts (Langourov, 2010).

* 127. Megaselia manicata (Wood, 1910) - Material examined: [N1.06] soil traps: 1.V-1.VI.1999, $3 \hat{\delta}$. Westpalaearctic species, known from Europe, North Africa (Tunisia) and Macaronesia. For the Balkan Peninsula known from Slovenia, Montenegro (Schmitz, 1928) and North Macedonia (Coe, 1956).

128. Megaselia meconicera (Speiser, 1925) - Ma-

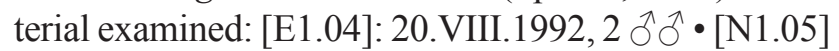
Malaise trap: 5-12.VI.1998, 1 ठ $\bullet$ [N1.06]:

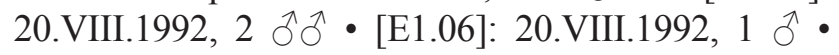
[N1.07]: 20.VIII.1992, 1 o $\cdot$ [E2.01], tree traps:

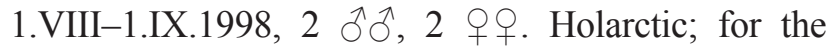
Balkan Peninsula known from Albania (Schmitz, 1928) and Montenegro (Langourov, 2004a). Other localities in Bulgaria: E Rhodope Mts (Langourov, 2004b) and W Rhodope Mts (Langourov, 2010).

* 129. Megaselia melanocephala (von Roser, 1840) - Material examined: [S1.01] Malaise trap:

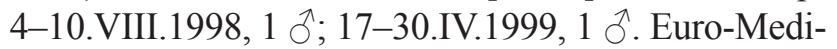
terranean; known from Middle and South Europe, North Africa (Tunisia) and Asia (Israel). For the Balkan Peninsula known from Croatia (Schmitz, 1924).

130. Megaselia nigriceps (Loew, 1866) - Material examined: [N1.04]: 25.IX.1992, 1 त $\bullet$ [E1.05]: 1.VII-1.VIII.1998, 1 q, tree traps; 1.IX-1.X.1998,

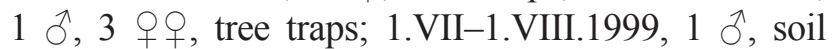

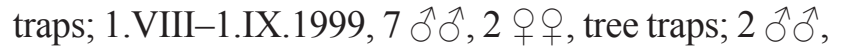

soil traps $\bullet$ between [E1.05] to [E1.06]: 27.VIII.1992, 1

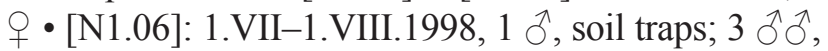
2 우우, tree traps; 1.VIII-1.IX.1998, 1 O , 1 우, soil traps; $13 \widehat{\partial} \hat{\jmath}$, tree traps; 1.VI-1.VII.1999, 1 ㅇ, tree traps; 1.VII-1.VIII.1999, 1 ô, soil traps; 1.VIII-1.IX.1999, 1 ठ, tree traps $\bullet$ between [E2.01] to [E2.03]: 20.VIII.1992, 1 § • [E2.01]: 20.VIII.1992, 1 P; 27.VIII.1992, 1 oे; 1.VIII-1.IX.1998, 1 ô, tree traps; 1.IX-1.X.1998, 2 ठิ่, tree traps;

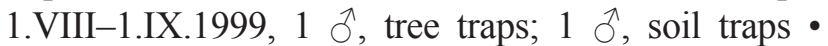
between [E2.01] to [E2.02]: 27.VIII.1992, 1 ऊ; 3.IX.1992, $1 \hat{\delta} \cdot[\mathrm{N} 2.02]:$ 3.IX.1992, $1 \hat{\jmath} \cdot$ between [N2.02] to [N2.03]: 27.VIII.1992, $1 \hat{0} \cdot$ [N2.03]: 20.VIII.1992, 1 o 1830 m, Bistrishko Branishte Reserve, near Fizkulturnik Chalet, subalpine zone (leg. V. Beschovski): 8.X.1981, 1 ๙ै $1840 \mathrm{~m}$, near Aleko Chalet, spruce wood: 12.IX.1999, 1 ठै feeding on sugar syrup. Holarctic; known from Europe, Asia (Japan, Russian Far East) and North America. Reported for the Balkan Peninsula as Megaselia projecta (Becker) from Croatia (Schmitz, 1924), Bulgaria (Schmitz, 1953a, without locality) and Montenegro (Langourov, 2004a).

** 131. Megaselia nigripalpis (Lundbeck, 1920) Material examined: [N1.04]: 30.V.1993, 1 กे • 1260-1360 m, near Esperanto Chalet, mixed wood: 30.V.1993, 1 ठ • [E3.02]: 1.VIII-1.IX.1999, 3 ふぇ, tree traps. European species, till now known from Germany, Denmark, Norway and Finland. New region: Balkan Peninsula - Bulgaria.

** 132. Megaselia obscuripennis (Wood, 1909) Material examined: [N1.05] 1150 m: 21.VII.1992, 1 ते - [E1.05] soil traps: 1.VIII-1.IX.1998, $1 \hat{0} \cdot[\mathrm{N} 1.05]$ Malaise trap: 5-12.VI.1998, 2 ô, 1 ㅇ $\cdot$ [N3.02]: 12.VI-4.VII.1999, 1 §. European species, known from Middle and North Europe. New region: Balkan Peninsula - Bulgaria.

133. Megaselia oxybelorum Schmitz, 1928 - Material examined: $1340 \mathrm{~m}$, near Kopitoto Peak, beech wood (leg. S. Lazarov): 5.VI.2000, 1 ภ $[$ [E3.02], soil traps: 1.VII-1.VIII.1999, 1 §. Westpalaearctic species, known from Southern Europe, Macaronesia, Dagestan (Caucasus) and Asia (Israel, Iran). Reported for the Balkan Peninsula from Bulgaria under the synonym Megaselia insecta Schmitz, 1953 (Schmitz, 1953a).

** 134. Megaselia palmeni (Becker, 1901) - Material examined: [E2.01], tree traps: 1.VI-1.VII.1999, 1 o $1840 \mathrm{~m}$, near Aleko Chalet, spruce wood (leg. V.

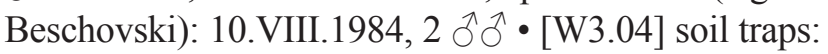

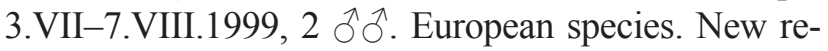
gion: Balkan Peninsula - Bulgaria. 
** 135. Megaselia pectinifera Schmitz, 1926 Material examined: [E3.02], soil traps: 1.X-1.XI.1998,

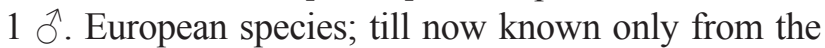
Alps (Austria and Switzerland). New region: Balkan Peninsula - Bulgaria.

136. Megaselia pectoralis (Wood, 1910) - Material examined: [E1.05], tree traps: 1.VII-1.VIII.1999, 1 ๙ • [N1.05] Malaise trap: 12-14.VI.1998, 1 ๙ • [E3.02]:

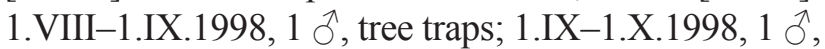
soil traps; 1.VI-1.VII.1999, 2 $\hat{\jmath}$, soil traps • [W3.04] soil traps: 3.VII-7.VIII.1999, 2 ठิ઼ • [S4.01]: 6.VII-7.VIII.1999, 3 ठิَ̄ • [W3.05] soil traps:

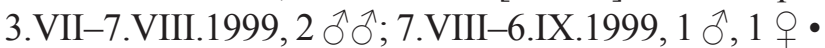
[E3.04] soil traps: 1.VII-1.VIII.1999, 2 ふふふぇ;

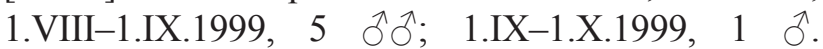
European species, for the Balkan Peninsula known from Montenegro (Langourov, 2004a). Other localities in Bulgaria: Sofia Plain (Langourov, 2004b) and Western Rhodope Mts (Langourov, 2010).

** 137. Megaselia pectorella Schmitz, 1929 - Material examined: [E2.01], tree traps: 1.VII-1.VIII.1999, $2 \widehat{\jmath} \widehat{\jmath}$. European species, known till now from Austria, Germany, Poland and Norway. New region: Balkan Peninsula - Bulgaria.

** 138. Megaselia perfusca Schmitz, 1935 - Material examined: [E2.01], soil traps: 1.VII-1.VIII.1999, $1 \hat{o} \cdot[\mathrm{W} 3.05]$ soil traps: 7.VIII-6.IX.1999, 1 ぇ $\bullet$ [E3.04] soil traps: 1.VII-1.VIII.1999, 2 $ぇ \hat{\jmath}$. European; known from Austria, Germany, Poland and Czech Republic. New region: Balkan Peninsula - Bulgaria.

* 139. Megaselia piliventris Schmitz, 1937 - Material examined: [N1.05] Malaise trap: 12-14.VI.1998, $1 \hat{\sigma} \cdot[\mathrm{E} 2.01]$, tree traps: 1.V-1.VI.1999, 32 ふぇ;

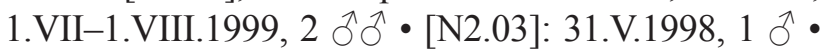
$1840 \mathrm{~m}$, near Aleko Chalet, spruce wood (leg. V. Beschovski): 10.VIII.1984, 1 ๙ • [N3.01]: 3.IX.1992, $1 \hat{\sigma} \cdot[\mathrm{E} 3.02]$, tree traps: 1.V-1.VI.1999, $1 \hat{\delta}$. Westpalaearctic species, known from Europe and Asia (Yemen). Reported for the Balkan Peninsula from North Macedonia (Coe, 1956; Langourov, 1999 as M. cirriventris Schmitz, 1929).

140. Megaselia pleuralis (Wood, 1909) - Material examined: [S1.01] Malaise trap: 18-28.VIII.1998,

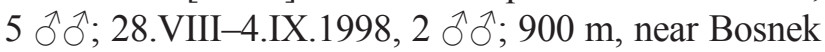
Village, near Popov Izvor Karst spring, MSS traps: 6.VI-2.X.2013, 1 §’; 910 m, near Bosnek Village, Popov Izvor Cave, soil traps: 2.X-2.XI.2013, 2 우; 954 m, near Bosnek Village, near Academik Cave, MSS traps: 6.VI-2.X.2013, 2 우 1000 m, above Bistritsa village, Formica pratensis Retzius, 1783 nest (leg. A. Lapeva): 14.XI.1997, 1 ๆ [E1.05]: 1.VIII-1.IX.1998, 12 우, tree traps; 40 우, soil traps; 1.IX-1.X.1998, 3 o o, tree traps; 1.VII-1.VIII.1999, 6 qq , soil traps; 1.VIII-1.IX.1999, 6 우, soil traps; 1.IX-1.X.1999, 1 q, tree traps; $1 \hat{\sigma}$, soil traps • [N1.06]: 1.VII-1.VIII.1998, 3 ఏิす, 27 우, soil traps; 2 우, tree traps; 1.VIII-1.IX.1998, 2 ๙઼, 11 우, soil

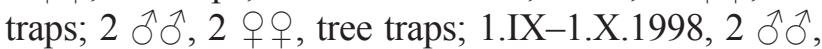
1 q, tree traps; 1.V-1.VI.1999, $2 \hat{\jmath} \widehat{\jmath}$, soil traps; 1.VIII-1.IX.1999, $1 \hat{\jmath}$, soil traps; 1.IX-1.X.1999, $1 \hat{\jmath}$, soil traps - [E2.01]: 1.VIII-1.IX.1998, 21 우, tree

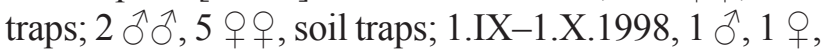
tree traps; 1.VII-1.VIII.1999, $2 \hat{\jmath}$, tree traps; 1.VIII-1.IX.1999, $1 \hat{\jmath}$, tree traps; 1.IX-1.X.1999, $1 \hat{\jmath}$, 1 O, soil traps $\bullet 1750$ m, near Kumata Chalet, spruce wood (leg. T. Ljubomirov): 22.VII.1997, 1 ๙ै 1840 m, near Aleko Chalet, spruce wood (leg. V. Beschovski):

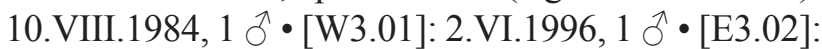

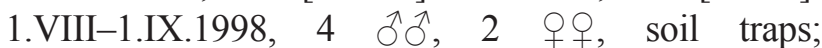
1.IX-1.X.1998, $1 \hat{\jmath}$, soil traps; 1.V-1.VI.1999, 1 ठ, soil traps; 1.VI-1.VII.1999, 1 ô, 1 q, tree traps; 1 q, soil traps; 1.VII-1.VIII.1999, 4 ๙ิे, 2 우, soil traps. Subcosmopolitan species with Holarctic origin, widespread in the country (Czerny, 1930; Langourov, 2001, 2004c, 2010). For the Balkan Peninsula also known from North Macedonia (Coe, 1956; Langourov, 1999), Croatia, Bosnia-Herzegovina (Schmitz, 1919; Schmitz, 1928; Schmitz, 1953; Schmitz, 1957) and Montenegro (Langourov, 2004a).

141. Megaselia plurispinulosa (Zetterstedt, 1860) - Material examined: [N1.01] Malaise trap:

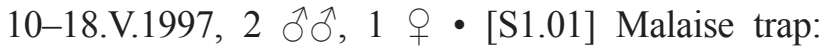
18-28.VIII.1998, 1 ふँ; 28.VIII-4.IX.1998, 1 ๙ •

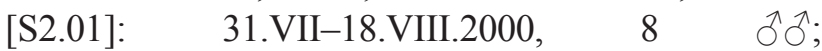
18.VIII-1.IX.2000, 5 ठ઼. Palaearctic species, known from Europe, Macaronesia and Asia (Turkey, Iran, China). For the Balkan Peninsula also known from Greece, Slovenia, Italy (Schmitz, 1924, Schmitz, 1928) and North Macedonia (Coe, 1956; Langourov, 1991). In Bulgaria: Sofia Plain (Langourov, 2004b).

142. Megaselia posticata (Strobl, 1898) - Material examined: [N1.01] Malaise trap: 10-18.V.1997, 1 ふ̋; 900 m, near Bosnek Village, near Popov Izvor Karst spring, MSS traps: 6.VI-2.X.2013, 1 ふ̄, 3 우; 1118 m, 992 m, near Bosnek Village, near Duhlata Cave, MSS traps: 18.IV-16.VI.2007, 2 ふึ. Westpalaearctic species, described from Croatia - Dubrovnik (Strobl, 1898); also known from Bosnia-Herzegovina, Slovenia (Schmitz, 1928). Other localities in Bulgaria: southern part of Vitosha Mt (Langourov et al., 2014). 
143. Megaselia producta (Schmitz, 1921) - Material examined: $1840 \mathrm{~m}$, near Aleko Chalet, spruce wood (leg. V. Beschovski): 10.VIII.1984, 2 ठิ 6.VIII-8.IX.1999, 1 ๙ • [E3.02]: 1.VIII-1.IX.1999, $1 \hat{\jmath}$, tree traps; 1 ô, soil traps - [E3.04] soil traps: 1.X-1.XI.1999, 1 ô, 1 q. Westernpalaearctic species, known from Europe and Asia (Iran). Other localities in Bulgaria: Eastern Rhodope Mountains (Langourov, 2004b) and Western Rhodope Mts (Langourov, 2010).

144. Megaselia propinqua (Wood, 1909) - Material examined: [E1.05] soil traps: 1.VIII-1.IX.1999, 2 ठิ઼ • [N1.06]: 1.VII-1.VIII.1998, $1 \hat{\jmath}$, soil traps; 1.V-1.VI.1999, 1 ふ, soil traps; 1.VI-1.VII.1999, $2 \hat{\jmath} \hat{\sigma}$, tree traps; 1.IX-1.X.1999, $1 \hat{\jmath}$, soil traps • [E2.01], soil traps: 1.IX-1.X.1999, 1 ô $\cdot$ [W3.02] soil traps: 3.VII-7.VIII.1999, 1 ふै $\cdot$ [E3.04] soil traps: 1.VII-1.VIII.1999, 1 $\widehat{\jmath}$. European; for the Balkan Peninsula known from Serbia (Schmitz, 1924). Other localities in Bulgaria: W Stara Planina (Schmitz, 1953).

** 145. Megaselia protarsalis Schmitz, 1927 Material examined: [N1.06] soil traps:

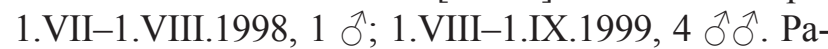
laearctic; from Middle Europe and Asia (Russian Far East). New region: Balkan Peninsula - Bulgaria.

* 146. Megaselia pseudogiraudii (Schmitz, 1920) - Material examined: [S2.01]: 23-30.V.2000, 1 ○ • [E1.05], tree traps: 1.VIII-1.IX.1998, 1 q. Euro-Mediterranean species, for the Balkan Peninsula known from Croatia (Schmitz, 1928).

147. Megaselia pumila (Meigen, 1830) - Material examined: $1100 \mathrm{~m}$, above Zheleznitsa, mixed wood (leg. B. Dimitrova): 27.V.1983, 1 ^ [N1.05] Malaise trap: 12-14.VI.1998, $2 \hat{\jmath} \cdot[\mathrm{N} 1.06]$ soil traps: 1.VII-1.VIII.1998, 1 ^ • [N1.07]: 20.VIII.1992, 1 • [E2.01]: 21.V.1997, $1 \hat{\jmath}, 1$, in copula; 1.VIII-1.IX.1999, $1 \hat{\jmath}$, tree traps; $1 \hat{\jmath}$, soil traps • [E2.03]: 20.VIII.1992, 1 q $\quad$ [N3.02]: 12.VI-4.VII.1999, 1 ๙ $1860 \mathrm{~m}$, Torfeno Branishte reserve - Kapaklivets place, peatbog in subalpine zone:

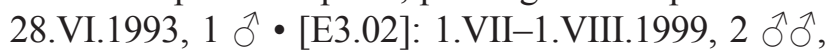

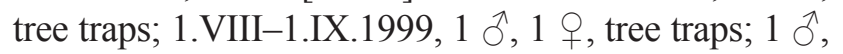
soil traps - [W3.02] soil traps: 5.VI-3.VII.1999, 1 ô, 1 q. Palaearctic; known from Europe, North Africa (Tunisia) and Asia (Israel, Russian Far East). Balkan Peninsula - Bosnia-Herzegovina (Strobl, 1898: as Phora pulicaria var. pumila), Croatia (Strobl, 1902: as Phora pulicaria var. pumila; Schmitz, 1924: as Aphiochaeta atripes Brues), Italy (Schmitz, 1928), North Macedonia (Langourov, 1999), Montenegro (Langourov, 2004a). Other localities in Bulgaria: Kresna
Gorge (Langourov \& Sakalian, 2001) and Western Rhodope Mts (Langourov, 2010).

148. Megaselia pusilla (Meigen, 1830) - Material examined: [N1.01] Malaise trap: 10-18.V.1997, 3 ठ઼ ठ - [S1.01] Malaise trap: 18-28.VIII.1998, 165 ふ઼ふ;

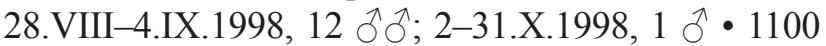
$\mathrm{m}$, above Zheleznitsa Village, coniferous plantation (legV. Beschovski): 18.V.1982, 1 ô [N1.05] $1150 \mathrm{~m}$ : 21.VII.1992, 1 त $\bullet$ [N1.05] Malaise trap: 5-12.VI.1998, 1 ふै [N1.06]: 1.VII-1.VIII.1998, 3

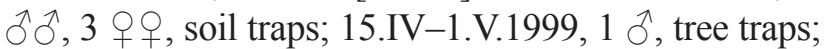

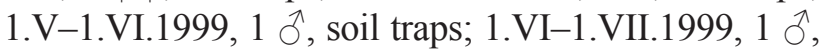
soil traps $\bullet 1118$ m, near Bosnek Village, Zhivata Voda Cave, 27.XI.2002, 3 우; [E3.02]: 1.V-1.VI.1999,

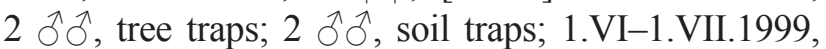
$1 \hat{\jmath}$, tree traps; $17 \hat{\jmath} \hat{\jmath}$, soil traps; 1.VII-1.VIII.1999, $4 \hat{\jmath} \bar{\jmath}$, tree traps $\bullet[\mathrm{N} 3.03]$ soil traps: 6.VIII-8.IX.1999,

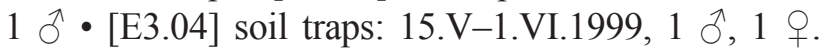
Holarctic species, known from Europe, Asia (China) and North America. For the Balkan Peninsula known from Slovenia (Schmitz, 1928) and Serbia (Coe, 1956). Other localities in Bulgaria: Kresna Gorge (Langourov \& Sakalian, 2001), Eastern Rhodope Mts (Langourov, 2004b), Western Rhodope Mts (Langourov, 2010), southern part of Vitosha Mt (Langourov et al., 2014).

* 149. Megaselia pygmaea (Zetterstedt, 1848) Material examined: [E1.01]: 15.IV.2000, 1 त・[E1.05], tree traps: 1.VIII-1.IX.1998, 1 9. European species, on the Balkan Peninsula known from Serbia and Slovenia (Schmitz, 1924; Schmitz, 1928).

** 150. Megaselia robusta Schmitz, 1928 - Material examined: [E3.02], tree traps: 1.V-1.VI.1999, 1 $\lesssim$. Holarctic; known from North Europe and North America. New region: Balkan Peninsula - Bulgaria.

151. Megaselia rubella (Schmitz, 1920) - Material

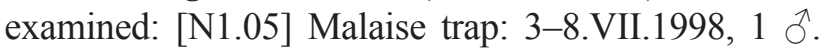
European species. Other localities in Bulgaria: Western Rhodope Mts (Langourov, 2010).

151. Megaselia ruficornis (Meigen, 1830) - Material examined: [N1.01] Malaise trap: 10-18.V.1997, 1 ऽ - [S1.01] Malaise trap: 18-28.VIII.1998, 2 ふึે, 1 q; $1120 \mathrm{~m}$, above Bosnek Village, on the road to Chuipetlyovo, scree in mixed wood, MSS traps: 6.VI-2.X.2013, 3 ๙ิळ ・ [E1.05], tree traps: 1.VIII-1.IX.1998, 1 q. Holarctic species, known from Europe, Macaronesia, Asia (Russian Far East) and North America. For the Balkan Peninsula is known from Croatia (Langhoffer, 1919; Schmitz, 1924; Coe, 1956) and Montenegro (Langourov, 2004a). Other localities in Bulgaria: W Rhodope Mts (Langourov, 2010). 
152. Megaselia rufipes (Meigen, 1804) - Material examined: [N1.01] Malaise trap: 10-18.V.1997, 1 §. [S1.01] Malaise trap: 4-10.VIII.1998, 2 ふふふ; 18-28.VIII.1998, 2 ふぇં; 2-31.X.1998, 1 ઠ. Cosmopolitan; widespread in the country. Balkan Peninsula Italy (Funk \& Graffe, 1895; Schmitz, 1919), BosniaHerzegovina (Strobl, 1900; Bezzi, 1911; Schmitz, 1919; Schmitz, 1928), Croatia (Strobl, 1902; Schmitz, 1919; Langhoffer, 1919; Schmitz, 1928; Coe, 1956), Montenegro (Schmitz, 1919), Greece - Corfu Island (Schmitz, 1924), North Macedonia (Langourov, 1999), Romania (Langourov, 2001).

** 153. Megaselia rupestris Schmitz, 1934 - Material examined: [E1.05] soil traps: 1.IX-1.X.1998, $3 \hat{\jmath}$. European species, known till now from Austria, Great Britain, Czech Republic and Spain. New region: Balkan Peninsula - Bulgaria.

154. Megaselia scalaris (Loew, 1866) - Material examined: 900 m, near Bosnek Village, near Popov Izvor Karst spring, MSS traps: 6.VI-2.X.2013, 7 ఓఓఓ, 1 q; 2.X-2.XI.2013, 1 o. Cosmopolitan; the only species of scuttle fly reported from Antarctica. Balkan Peninsula - Greece and Serbia (Langourov, 2004b). In Bulgaria: Sofia Plain (Langourov, 2004b).

155. Megaselia scutellaris (Wood, 1909) - Material examined: [S1.01] Malaise trap: 2-31.X.1998, 1 ○ •

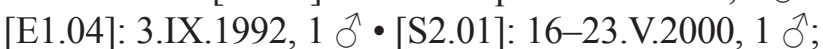
8-31.X.2000, 2 ठえへ・[E1.05]: 1.VIII-1.IX.1998, 1 ठे, tree traps; 1.IX-1.X.1998, $1 \hat{\sigma}, 1$, tree traps; $2 \hat{\sigma} \widehat{\jmath}$, soil traps; 1.V-1.VI.1999, 1 \&, tree traps; 1.VII-1.VIII.1999, 1 ふ, 2 o, 1.VIII-1.IX.1999, $4 \hat{\jmath} \hat{\jmath}, 1$ o, tree traps; 1.IX-1.X.1999, 5 우, soil traps; 1.X-1.XI.1999, 4 oq, soil traps - [N1.06] soil traps: 1.VII-1.VIII.1999, $1 \hat{\jmath} \cdot$ [E2.01], soil traps: 1.VIII-1.IX.1998, 1 ふ;; 1.IX-1.X.1999, 1 ふ;

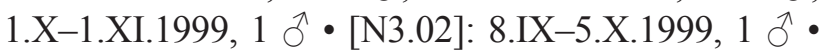
[E3.02], soil traps: 1.VII-1.VIII.1999, 1 ふँ;

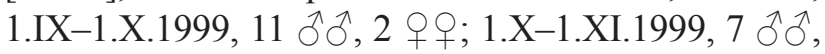
1 - [E3.04] soil traps: 1.VII-1.VIII.1999, 1 ふ̋; 1.IX-1.X.1999, 1 ふ̋. European; Balkan Peninsula Bosnia-Herzegovina (Schmitz, 1919), Croatia (Schmitz, 1924), Albania, Slovenia (Schmitz, 1928). In Bulgaria: Eastern Rhodope Mts (Langourov, 2004b) and Western Rhodope Mts (Langourov, 2010).

** 156. Megaselia sepulchralis (Lundbeck, 1920) - Material examined: [E2.01], tree traps:

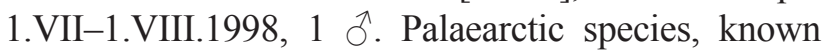
from North and Middle Europe and Asia (Russian Far East). New region: Balkan Peninsula - Bulgaria.
** 157. Megaselia sericata Schmitz, 1935 - Mater-

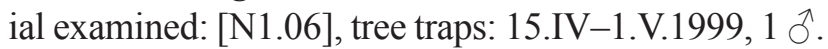
European; known till now only from Austria and Germany. New region: Balkan Peninsula - Bulgaria.

** 158. Megaselia setulipalpis Schmitz, 1938 Material examined: [E1.05], tree traps: 1.VII-1.VIII.1998, 1 §. European species, known from North and Middle Europe. New region: Balkan Peninsula - Bulgaria.

* 159. Megaselia simplex (Wood, 1910) - Material examined: [E1.03]: 15.IV.2000, 1 $\widehat{\jmath}$. European species. On the Balkan Peninsula also known from Montenegro (Langourov, 2004a).

160. Megaselia simulans (Wood, 1912) - Material examined: [N1.06] soil traps: 1.VIII-1.IX.1999, $1 \hat{\jmath}$. Westernpalaearctic species, known from Europe and Asia (Eastern Siberia). For the Balkan Peninsula also known from Croatia (Coe, 1956). Other localities in Bulgaria: Western Rhodope Mts (Langourov, 2010).

161. Megaselia sordida (Zetterstedt, 1838) - Material examined: $2226 \mathrm{~m}$, Skoparnik Peak, subalpine zone (leg. V. Beschovski): 21.VIII.1981, 2 ठ઼ đ. Holarctic species, known from Europe, Asia (Siberia, Russian Far East) and North America. Other localities in Bulgaria: Western Rhodope Mts (Langourov, 2010).

** 162. Megaselia spinata (Wood, 1910) - Material examined: [N1.06], tree traps: 1.VIII-1.IX.1999, 1 o. European species, known till now from Austria and Great Britain. New region: Balkan Peninsula Bulgaria.

* 163. Megaselia spinigera (Wood, 1908) - Material examined: [N1.01] Malaise trap: 12-17.V.1998, 1 ऽ - [N1.06] soil traps: 1.V-1.VI.1999, $1 \hat{\sigma} \cdot[\mathrm{E} 3.02]$ : 1.VII-1.VIII.1998, 1 ふ, tree traps; 1.V-1.VI.1999, $1 \delta$, soil traps. European species. For the Balkan Peninsula also known from Croatia (Schmitz, 1924).

164. Megaselia stichata (Lundbeck, 1920) - Material examined: [E1.05], tree traps: 1.VI-1.VII.1999, 1 ऽ. Westpalaearctic species, known from Europe, Macaronesia and Asia (Israel, Iran). For the Balkan Peninsula also known from Montenegro (Langourov, 2004a). Other localities in Bulgaria: Sofia Plain (Langourov, 2004b).

* 165. Megaselia styloprocta (Schmitz, 1921) Material examined: [E1.03]: 15.IV.2000, 1 ก $\bullet$ [N1.05] Malaise trap: 29.V-5.VI.1998, 1 ふ̋; 5-12.VI.1998, $3 \hat{\jmath} \hat{\sigma} \cdot[\mathrm{N} 1.06]$ soil traps: 1.V-1.VI.1999, $1 \hat{\jmath}$. Westpalaearctic species, known from Europe and Asia (Iran). For the Balkan Peninsula also known from Slovenia (Schmitz, 1928). 
** 166. Megaselia subconvexa (Lundbeck, 1920)Material examined: [S1.01] Malaise trap: 18-28.VIII.1998, 1 ๙ $\quad$ [N1.06], tree traps: 1.VI-1.VII.1998, 1 ふे. European species, known till now from Austria, Great Britain, Denmark and Netherlands. New region: Balkan Peninsula - Bulgaria.

* 167. Megaselia subpleuralis (Wood, 1909) - Material examined: [E1.05] soil traps: 1.VII-1.VIII.1999, $1 \hat{\sigma} \cdot[E 2.01]:$ 28.V.1998, 1 $\hat{\jmath}$. Holarctic species, known from Europe, Macaronesia, Asia (Israel) and North America. For the Balkan Peninsula also known from Montenegro (Langourov, 2004a). Other localities in Bulgaria: Western Rhodope Mts (Langourov, 2010).

168. Megaselia subtumida (Wood, 1909) - Material examined: [N1.06] soil traps: 1.VII-1.VIII.1998, 1 o; 1.VI-1.VII.1999, 1 o; 900 m, near Bosnek Village, near Popov Izvor Karst spring, MSS traps:

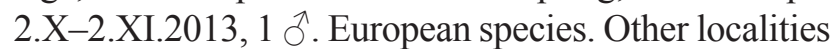
in Bulgaria: W Rhodope Mts (Langourov, 2010) and southern part of Vitosha Mt (Langourov et al., 2014).

169. Megaselia sylvatica (Wood, 1910) - Material examined: [N1.05] Malaise trap: 11-15.VII.1998, 1 శึ - [N1.06] soil traps: 1.IX-1.X.1999, 1 స̃. European species. For the Balkan Peninsula also known from Montenegro (Langourov, 2004a). Other localities in Bulgaria: North Black Sea Coast (Schmitz, 1953).

** 170. Megaselia tama (Schmitz, 1926) - Material examined: [N1.01] Malaise trap: 5-12.V.1998, 1 ^ • [S1.01] Malaise trap: 10-18.VIII.1998, 1 o. Westpalaearctic species, known from Europe and Asia (Iran). New region: Balkan Peninsula - Bulgaria.

* 171. Megaselia tarsalis (Wood, 1910) - Material examined: [N1.01] Malaise trap: 10-18.V.1997, 2 ठ઼ - [E1.05]: 1.VII-1.VIII.1999, 1 ô, tree traps;

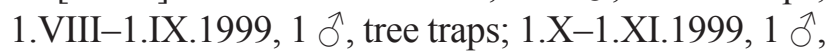
tree traps; 1 §, soil traps - [N1.06]: 2.X.1999, 1 §;

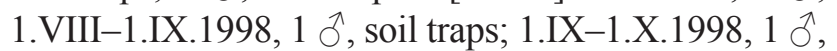
soil traps; 1.X-1.XI.1998, 1 ふै, soil traps; 1.VI-1.VII.1999, 1 §ै, soil traps • [E3.04] soil traps:

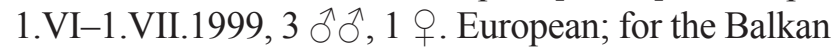
Peninsula is known from Greece (Schmitz, 1924), North Macedonia, Croatia (Coe, 1956; Langourov, 1999) and Montenegro (Langourov, 2004a).

172. Megaselia tarsella (Lundbeck, 1921) - Material examined: [S1.01] Malaise trap: 4-10.VIII.1998, 1 o $\cdot$ [N1.06] soil traps: 1.VIII-1.IX.1998, 1 ऊ; 1.V-1.VI.1999, 3 ふ઼ふ̄, 2 우 • 1840 m, near Aleko Chalet, spruce wood: 12.IX.1999, $1 \hat{\jmath}-$ feeding on sugar syrup • [E3.02], soil traps: 1.VII-1.VIII.1999, $1 \hat{\sigma} \cdot[\mathrm{E3} .04]$ soil traps: 1.VI-1.VII.1999, 20 ठิ઼,
5 우; 1.VII-1.VIII.1999, 20 ふึฎ, 9 우. Palaearctic species, known from Europe and Asia (Russian Far East). Other localities in Bulgaria: Sofia Plain (Langourov, 2004b).

** 173. Megaselia tarsicia Schmitz, 1926 - Material examined: [E1.05] soil traps: 1.IX-1.X.1998, 1 ^ • [E2.01]: 1.VII-1.VIII.1998, 4 ठิ઼, 5 우, tree traps;

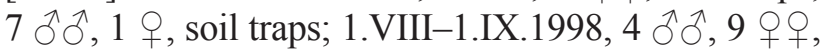
tree traps; 10 ठิ઼, 7 우, soil traps; 1.IX-1.X.1998,

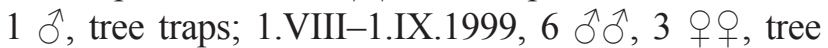
traps; $3 \hat{\jmath} \hat{\jmath}$, soil traps • [E3.02]: 1.VII-1.VIII.1998,

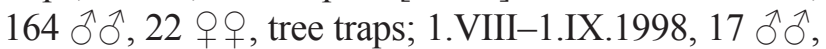
5 우, tree traps; 1.IX-1.X.1998, $1 \hat{\jmath}$, tree traps; 1.VI-1.VII.1999, $67 \hat{\jmath} \hat{\sigma}, 10$ oq, tree traps;

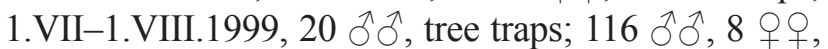
soil traps; 1.VIII-1.IX.1999, 2 ठิ̄, 3 oq, tree traps; 3 ふ઼,, 2 qo, soil traps - [W3.05] soil traps: 3.VII-7.VIII.1999, 1 $\lesssim$. Palaearctic species, known from Europe and Asia (Russian Far East). New region: Balkan Peninsula - Bulgaria.

174. Megaselia tenebricola Schmitz, 1934 - Material examined: $1006 \mathrm{~m}$, near Bosnek Village, near Duhlata Cave, MSS traps: 2.X-2.XI.2013, 1 సं; 1120 $\mathrm{m}$, above Bosnek Village, on the road to Chuipetlyovo, scree in mixed wood, MSS traps: 6.VI-2.X.2013, $1 \hat{\jmath}$. European species. Other localities in Bulgaria: ForeBalkan Mts and Vitosha (Langourov, 2001; Langourov et al., 2014).

175. Megaselia trichorrhoea (Schmitz, 1921) Material examined: [N1.06] soil traps: 1.VII-1.VIII.1998, 3 ふふ઼. European species. For the Balkan Peninsula also known from Croatia, North Macedonia (Coe, 1956) and Bulgaria (Disney, 1991).

* 176. Megaselia tumida (Wood, 1909) - Material examined: [E1.05] soil traps: 1.IX-1.X.1998, 1 q. Westpalaearctic species, known from Europe and North Africa (Tunisia). For the Balkan Peninsula also known from Bosnia-Herzegovina (Schmitz, 1919) and Croatia (Coe, 1956).

** 177. Megaselia uliginosa (Wood, 1909) - Material examined: [E1.05], tree traps: 1.VIII-1.IX.1999, 1 ऽ. European species, known from Middle Europe. New region: Balkan Peninsula - Bulgaria.

178. Megaselia unicolor (Schmitz, 1919) - Material examined: [E1.05], tree traps: 1.VIII-1.IX.1998, 1 o • [E2.01]: 1.VII-1.VIII.1998, 1 ô, soil traps; 1.VIII-1.IX.1999, 1 ô, tree traps - [E3.02]: 1.VIII-1.IX.1998, 9 ふ઼َ, 1 ㅇ, tree traps; 2 우, soil traps; 1.IX-1.X.1998, $1 \hat{\jmath}$, tree traps; 1 , , soil traps; 1.VII-1.VIII.1999, $49 \widehat{\jmath} \hat{\jmath}$, numerous + + + , tree traps; 


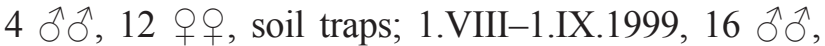

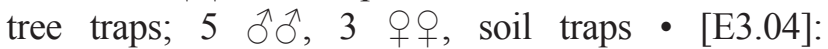

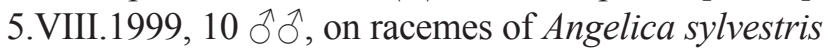
L. - [W3.03] soil traps: 3.VII-7.VIII.1999, 1 ๙ • [W3.05] soil traps: 3.VII-7.VIII.1999, 3 ठ $\sigma^{\Uparrow} \cdot[$ [E3.04] soil traps: 1.VIII-1.IX.1998, 1 गे, 3 우우;

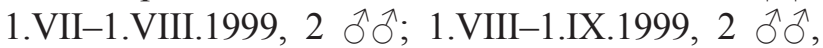
2 우우 1.XI.1999-1.V.2000, 1 $\widehat{\jmath}$. Palaearctic species, known from Europe and Asia (China). Other localities in Bulgaria: Eastern Rhodope Mts (Langourov, 2004c).

* 179. Megaselia variana Schmitz, 1926 - Material examined: [E1.05] soil traps: 1.VII-1.VIII.1999, 1 o ; 1.IX-1.X.1999, 1 o • [N1.05] Malaise trap: 12-14.VI.1998, 1 ठ․ European species. For the Balkan Peninsula known from Croatia (Schmitz, 1924).

180. Megaselia verna Schmitz, 1932 - Material ex-

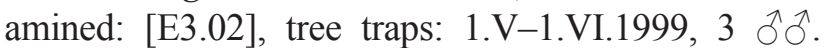
Euro-Mediterranean species. Other localities in Bulgaria: Kresna Gorge (Langourov \& Sakalian, 2001).

181. Megaselia vernalis (Wood, 1909) - Material examined: [W1.01] tree traps: 8-14.VII.1998, 1 ๙ $\bullet$ [E1.01]: 15.IV.2000, 1 ते $\cdot$ [E1.05] soil traps: 1.XI.1998-1.V.1999, 1 § , 1 o • [N1.06] soil traps: 15.IV-1.V.1999, 8 ठิす, 2 우; 1.V-1.VI.1999, 6 ठえ, 3 우우 1.VI-1.VII.1999, 2 ふふふ, 1 우 •[E2.01], tree traps: 1.VIII-1.IX.1999, 1 $\uparrow \cdot[\mathrm{E} 3.04]$ soil traps: $15 . \mathrm{V}-$ 1.VI.1999, 1 $\delta$. European species. Other localities in Bulgaria: Eastern Rhodope Mts (Langourov, 2004c).

182. Megaselia verralli (Wood, 1910) - Material examined: [S1.01] Malaise trap: 4-10.VIII.1998,

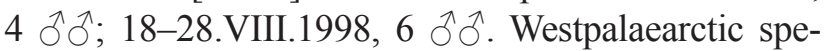
cies, known from Europe, Macaronesia and Asia (Israel, Iran). For the Balkan Peninsula also known from Slovenia, Greece (the island of Crete) (Schmitz, 1928) and North Macedonia (Coe, 1956). Other localities in Bulgaria: Sofia Plain, Sofia (Schmitz, 1953).

183. Megaselia vestita (Wood, 1914) - Material

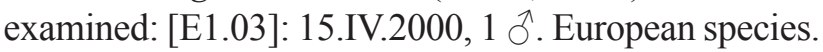
In Bulgaria: W Rhodope Mts (Langourov, 2010).

** 184. Megaselia woodi (Lundbeck, 1922) - Material examined: [S2.01]: 8-31.X.2000, 1 ठ • [E1.05]: 1.X-1.XI.1998, 1 Oे, 1 \% , tree traps; 1.X-1.XI.1999, 1 o, soil traps - between [E1.05] to [E1.06]: 17.X.1992, 3 के 0 • [N1.06] soil traps: 1.IX-1.X.1998, 1 ô, 1 q between [N1.07] to [N2.01]: 27.VIII.1992,

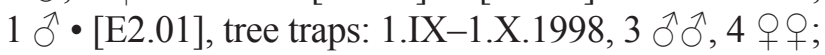
1.X-1.XI.1999, 1 ठै $1830 \mathrm{~m}$, Bistrishko Branishte Reserve, near Fizkulturnik Chalet, subalpine zone (leg. V. Beschovski): 8.X.1981, 1 กे • [E3.02]: 1.IX-1.X.1998, 1 ô, soil traps; 1.VIII-1.IX.1999,
6 ठぇ, tree traps; 1.IX-1.X.1999, 1 ठ, tree traps • [E3.03] soil traps: 8.IX-5.X.1999, 1 ㅇ $\cdot$ [E3.04] soil traps: 1.VIII-1.IX.1998, 1 ठో; 1.IX-1.X.1998, 1 హ;

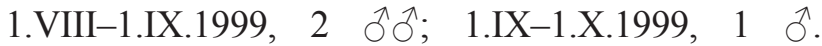
European. New region: Balkan Peninsula - Bulgaria.

185. Megaselia xanthozona (Strobl, 1892) - Material examined: [N1.01] Malaise trap: 10-18.V.1997, 1 ก $\cdot$ [S1.01] Malaise trap: 18-28.VIII.1998, 1 ơ; 28.VIII-4.IX.1998, 1 त $\bullet$ [E3.01] soil traps: 6.VI-3.VII.1999, 1 ô, 2 qq • [E3.02]: 1.VI-1.VII.1999, 1 §ิ, soil traps; 1.VII-1.VIII.1999, $1 \delta$, tree traps. Palaearctic; for the Balkan Peninsula also known from North Macedonia (Langourov, 1999). In Bulgaria: W Rhodope Mts (Langourov, 2010).

* 186. Menozziola schmitzi (Menozzi, 1921)-Material examined: [N1.05] Malaise trap: 19-26.VI.1998, $1 \delta$. Euro-Mediterranean species. For the Balkan Peninsula also known from Croatia (Schmitz, 1928).

187. Phalacrotophora fasciata (Fallen, 1823) Material examined: [S1.01] Malaise trap: 4-10.VIII.1998, 1 §. Palaearctic species, known from Europe and Asia (Turkey, Israel, Iran, China, Siberia, Russian Far East). For the Balkan Peninsula also known from Italy (Funk \& Graffe, 1895). Other localities in Bulgaria: Sofia Plain (Langourov, 2004b).

** 188. Plectanocnema nudipes (Becker, 1901) Material examined: [S1.01] Malaise trap: 3-17.IV.1999, 1 §. Holarctic species, known from Europe, Asia (Russian Far East) and North America. New region: Balkan Peninsula - Bulgaria.

** 189. Tubicera lichtwardti Schmitz, 1920 - Material examined: [S2.01]: 28.VI-15.VII.2000, 2 ठึ ठึ. Mediterranean species, known until now from Spain, France, Hungary and Israel. New region: Balkan Peninsula-Bulgaria.

190. Gymnophora arcuata (Meigen, 1830) - Material examined: [S1.01] Malaise trap: 18-28.VIII.1998, 1 क; 25.IX-2.X.1998, 1 ภ̊; 900 m, near Bosnek Village, near Popov Izvor Karst spring,

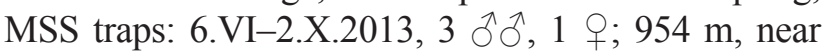
Bosnek Village, near Academik Cave, MSS traps: 6.VI-2.X.2013, 1 §. Westpalaearctic species, known from Europe and Asia (Israel, Iran). For the Balkan Peninsula also known from Italy (Funk \& Graffe, 1895), Bosnia-Herzegovina (Strobl, 1898), Croatia (Strobl, 1902, Langhoffer, 1919), Greece (Disney, 1991), North Macedonia (Langourov, 1999) and Montenegro (Langourov, 2004a). Other localities in Bulgaria: southern part of Vitosha Mt (Langourov et al., 2014). 
191. Gymnophora integralis Schmitz, 1920 - Material examined: [N1.01] Malaise trap: 10-18.V.1997,

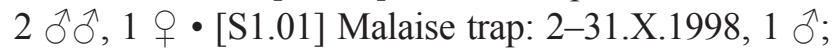

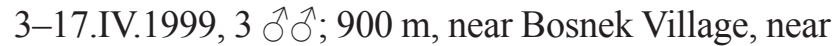
Popov Izvor Karst spring, MSS traps: 2.X-2.XI.2013,

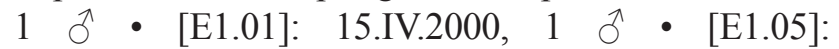
1.VI-1.VII.1998, 1 §’, soil traps; 1.IX-1.X.1998, 1 q, tree traps; 1 q, soil traps; 1.X-1.XI.1998, $1 \hat{\sigma}$, soil traps; 1.V-1.VI.1999, 1 ఏ, tree traps; $2 \widehat{\jmath} \hat{\jmath}$, soil traps; 1.IX-1.X.1999, 1 đ̃, 3 q, , soil traps • [N1.05] Malaise trap: 26.VI-3.VII.1998, 1 $\hat{\sigma}, 1$ ○ $[$ [N1.06] soil traps: 1.IX-1.X.1998, 1 ふ઼; 15.IV-1.V.1999, 1 ô, 1 o ; 1.V-1.VI.1999, 1 ふँ; 1.VI-1.VII.1999, 2 ふぇં; 1.IX-1.X.1999, 2 우; 1.X-1.XI.1999, $1 \hat{\jmath}$. Palaearctic species, known from Europe and Asia (Israel, Japan, Russian Far East). For the Balkan Peninsula it is known from Croatia (Schmitz, 1928). Other localities in Bulgaria: Eastern Rhodope Mts (Langourov, 2004c) and southern part of Vitosha Mt (Langourov et al., 2014).

** 192. Gymnophora quartomollis Schmitz, 1920 - Material examined: [E1.05]: 1.VI-1.VII.1999, 1 ふ,, 1 O, soil traps; 1.VII-1.VIII.1999, 1 o, tree traps; 7 ふิ઼, 3 우, soil traps; 1.VIII-1.IX.1999, 1 q, soil traps - [N1.06]: 1.VIII-1.IX.1998, 2 우, soil traps; 1.VI-1.VII.1999, 1 q, soil traps; 1.VII-1.VIII.1999, 4 우, soil traps; 1.VIII-1.IX.1999, 1 đ, 2 우, tree traps; 2 ठิす, 2 qq, soil traps - [E2.01], soil traps: 1.VIII-1.IX.1999, 1 §, 1 q. European species. New region: Balkan Peninsula - Bulgaria.

** 193. Metopina braueri (Strobl, 1880) - Material examined: [S1.01] Malaise trap: 4-10.VIII.1998, 3 ๙ิ઼, 2 우; 10-22.VII.1999, 3 qo . Westpalaearctic species, known from Europe and Asia (Israel). New region: Balkan Peninsula - Bulgaria.

** 194. Metopina crassinervis Schmitz, 1920 Material examined: [S1.01] Malaise trap:

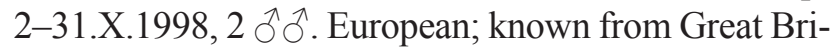
tain, Germany, Switzerland, Slovakia and Netherlands. New region: Balkan Peninsula - Bulgaria.

195. Metopina galeata (Haliday, 1833) - Material examined: [S1.01] Malaise trap: 4-10.VIII.1998, 4 ふふર, 5 qq; 10-22.VII.1999, 5 qq; $1006 \mathrm{~m}$, near Bosnek Village, near Duhlata Cave, MSS traps: 6.VI-2.X.2013, 1 9. European species. For the Balkan Peninsula also known from Greece (Disney, 1991) and Montenegro (Langourov, 2004a). Published for the mountain by Langourov et al. (2014).

* 196. Metopina heselhausi Schmitz, 1914 - Material examined: [S1.01] Malaise trap: 4-10.VIII.1998, 18 ふふ઼, 1 ○ [N1.06], tree traps: 1.VIII-1.IX.1999,
$1 q \cdot$ [E3.02], tree traps: 1.VII-1.VIII.1999, 1 §. Westpalaearctic species, known also from the Afrotropical Region (Benin). For the Balkan Peninsula also known from Greece (Disney, 1991).

* 197. Metopina oligoneura (Mik, 1867) - Material examined: [S1.01] Malaise trap: 4-10.VIII.1998,

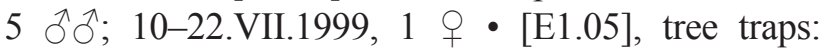
1.IX-1.X.1998, 1 ^ $\bullet$ [W3.03] soil traps: 3.VII-7.VIII.1999, 1 q. Westpalaearctic; known from Europe and Asia (Iran). For the Balkan Peninsula it is known from Montenegro (Langourov, 2004a).

* 198. Metopina perpusilla (Six, 1878) - Material examined: [N1.02] Malaise trap: 10-17.VIII.1999, 2 우 - [S1.01] Malaise trap: 4-10.VIII.1998, 1 ․ European species, for the Balkan Peninsula it is known from Croatia (Schmitz, 1928).

* 199. Metopina pileata Schmitz, 1936 - Material examined: [N1.02]: 27.X.1999, $1 \hat{\sigma} \cdot[N 1.02]$ Malaise trap: 1-18.IX.1999, 3 우 - [S1.01] Malaise trap: 4-10.VIII.1998, 8 ふふふ; 10-22.VII.1999, 2 qq • [S2.01]: 31.VII-18.VIII.2000, 1 ○ [N1.06], tree traps: 1.IX-1.X.1999, 1 q. Westpalaearctic; known from Europe, North Africa (Algeria) and Asia (Israel). Balkan Peninsula - Greece (Disney, 1991).

** 200. Metopina ulrichi Disney, 1979 - Material examined: [S1.01] Malaise trap: 4-10.VIII.1998, 1 q; 10-22.VII.1999, 6 우. Westpalaearctic species, known from Europe and Asia (Israel). New region: Balkan Peninsula - Bulgaria.

201. Beckerina umbrimargo (Becker, 1901) - Material examined: [N1.01] Malaise trap: 10-18.V.1997,

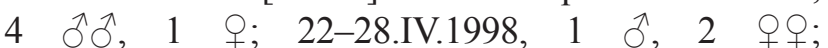

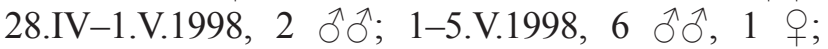

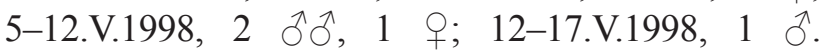
European species. Other localities in Bulgaria: Western Rhodope Mts (Langourov, 2010).

\section{Discussion}

Two hundred and one species, belonging to 19 genera as follows - Aenigmatias (2), Anevrina (4), Beckerina (1), Borophaga (1), Chaetopleurophora (4), Conicera (5), Diplonevra (6), Gymnophora (3), Gymnoptera (1), Megaselia (125), Menozziola (1), Metopina (8), Peromitra (2), Phalacrotophora (1), Phora (10), Plectanocnema (1), Spiniphora (4), Triphleba (21), Tubicera (1) - have been established on Vitosha during the investigation. Gymnoptera, Peromitra, Plectanocnema and Tubicera are recorded for the first time for 
the Balkan Peninsula and Menozziola - for Bulgaria. Sixty-nine species are new for the Balkan Peninsula and additional 32 - for Bulgaria. For most of these species the Balkan Peninsula is the southernmost part of their known range. Of special interest among them are Triphleba longifurcata, T. withersi and Megaselia abernethae, known until now only from their typelocalities. On the basis of the established number of species, it can be concluded that Vitosha is an area with a significant scuttle fly diversity.

Five plant species, belonging to four families, visited by the scuttle flies for additional nutrition were identified during the research. They are as follows: Iridaceae, Crocus veluchensis Herb. (Triphleba intermedia and T. pachyneurella); Salicaceae, Salix caprea L. (Triphleba intermedia); Apiaceae, Angelica sylvestris L. (Megaselia aculeata, M. coei, M. unicolor, Phora stictica, Spiniphora jugorum) and Anthriscus nitida (Wahlenb.) Garcke (Phora tincta); Asteraceae, Petasites hybridus ochroleucus (Boiss. \& A. Huet) Šourek (Conicera floricola, Megaselia angusta). All the above mentioned species of scuttle flies (with the exception of M. coei) are recorded for the first time as flower visitors of these plants. Flower visiting by scuttle flies of family Iridaceae and three of the species (excluding A. sylvestris and Salix caprea) is recorded for the first time. For four scuttle fly species ( $T$. pachyneurella, Megaselia aculeata, M. unicolor and M. angusta) anthophilia is recorded for the first time.

After the present investigation, the number of the established scuttle flies for Bulgaria increases to 231, while for the Balkan Peninsula it is 279 (Schmitz, 1924, 1928, 1953; Disney, 1991, 2003; Langourov, 1999, 2001, 2004a, 2004b, 2004c, 2010; Langourov \& Sakalian, 2001; Langourov et al., 2014).

\section{Acknowledgments}

I would like to thank all my colleagues who kindly submitted the materials collected by them - O. Mikov, V. Beschovski, T. Ljubomirov, E. Manasieva, S. Lazarov, N. Simov, A. Lapeva, B. Dimitrova.

\section{References}

Becker T. 1901 Die Phoriden. Abhandlungen der Zoologisch-Botanischen Gesellschaft in Wien 1: $1-100$.
Beschovski V., Langourov M. 1997 Contribution to the study of the distribution of Phoridae species in the Balkan Peninsula (Diptera, Phoridae). Acta zoologica bulgarica 49: 43-47.

Bezzi M. 1911 Biospeleologica. 20. Diptères (Première série). Archives de zoologie expérimentale et générale 8: 1-87.

Coe R. 1956 Diptera taken in Yugoslavia from May to July, 1955, with localities and notes. Bulletin du Muséum d'Histoire Naturelle du Pays Serbe, Belgrade, B 8: 75-96.

Colyer C.N. 1956 Two new species of Megaselia (Diptera, Phoridae) from Yugoslavia. Bulletin du Muséum d'Histoire Naturelle du Pays Serbe, Belgrade, B 8: 97-100.

Czerny L. 1930 Dipteren auf Schnee und in Höhlen. Mitteilungen aus den Königlichen naturwissenschaftlichen Instituten in Sofia - Bulgarien 3: 113-118.

Disney R.H.L. 1991 Family Phoridae. In: Soos A. (ed.) Catalogue of Palearctic Diptera, vol. 7: 143-204.

Disney R.H.L. 2003 Revisionary notes on European Phoridae (Diptera). Bonner zoologische Beiträge 50 (4): 293-304.

Funk A., Graffe E. 1895 Contributo alla fauna dei Ditteri dei dintorni di Trieste. Atti del Museo civico di storia naturale di Trieste 9 (3 n.s.): 211-234.

Hazelton M. 1970 Fauna from some caves in Bulgaria and one in Yugoslavia. The Transactions Cave Research Group of Gr. Britain, Ledbury 12 (1): 33-37.

Langhoffer A. 1919 Beiträge zur Dipterenfauna Kroatiens (Fortsetzung). Glasnik Hrvastkoga prirodoslovnoga društva 31 (1-4): 125-139.

Langourov M. 1999 Review on scuttle flies of Macedonia (Diptera: Phoridae). Proceedings of the I Congress of Ecologists of the Republic of Macedonia with International Participation. Special issues of Macedonian Ecological Society 5 (1): 303-312.

Langourov M. 2001 Scuttle flies from caves in the Balkan Peninsula (Diptera: Phoridae). Acta zoologica bulgarica 53 (3): 33-40.

Langourov M. 2004a Scuttle flies (Diptera: Phoridae) from Biogradska Gora National Park Montenegro. In: Pesic V., Spalevic V., Karaman G., Jovovic Z., Langourov M. (eds) The Biodiversity of the Biogradska Gora National Park. Monographies I, Department of Biology, University of Montenegro and Centre for Biodiversity of Montenegro: 104-112. 
Langourov M. 2004b Scuttle flies (Diptera: Phoridae) from Urban and Suburban Areas in the Sofia Plain. In: Penev L., Niemela J., Kotze J., Chipev N. (eds) Ecology of the City of Sofia. Species and Communities in an Urban Environment. Pensoft Publishers, Sofia-Moscow: 429-436.

Langourov M. 2004c Scuttle flies (Diptera: Phoridae) from Eastern Rhodopes (Bulgaria) with special consideration of winter-active species. In: Beron P., Popov A. (eds) Biodiversity of Bulgaria. 2. Biodiversity of Eastern Rhodopes (Bulgaria and Greece). Pensoft and National Museum of Natural History, Sofia: 759-768.

Langourov M. 2009 A New to Southeastern Europe Subfamily of Scuttle Flies (Diptera: Phoridae). Acta zoologica bulgarica 61 (1): 97-100.

Langourov M. 2010 Some scuttle flies (Diptera: Phoridae) from the Western Rhodope Mountains. In: Beron P. (ed.) Biodiversity of Bulgaria. 4. Biodiversity of Western Rhodopes (Bulgaria and Greece). Pensoft and National Museum of Natural History, Sofia: 485-498.

Langourov M., Lazarov S., Stoev P., Guéorguiev B., Deltshev C., Petrov B., Andreev S., Simov N., Bekchiev R., Antonova V., Ljubomirov T., Dedov I., Georgiev D. 2014 New and interesting records of the MSS and cave fauna of Vitosha Mt, Bulgaria. Balkan Speleological Conference "Sofia'2014" 2014: 66-76.

Langourov M., Sakalian V. 2001 Scuttle flies (Diptera: Phoridae) from Kresna Gorge (SW Bulgaria). In: Beron, P. (ed.): Biodiversity of Kresna Gorge (SW Bulgaria): 217-218. (In Bulgarian)

Pax F. 1937 Beobachtungen über das Tierleben einer istrischen Trockenhöhle. Mitteilungen über Höhlen- und Karstforschung. Berlin 1937 (2/3): $88-95$.

Schmitz H. 1919 Die Phoridenfauna der von Dr. Karl Absolon 1908-1918 besuchten mittel- und südosteuropäischen Höhlen. Tijdschrift voor Entomologie. Amsterdam 61: 232-241.
Schmitz H. 1924 Europäische Phoriden des Ungarischen National-Museums. Annales historiconaturales Musei nationalis hungarici, Budapest 21: 79-86.

Schmitz H. 1928 Die geographische Verbreitung der europäischen Phoridenarten. Natuurhistorisch Maandblad. Maastricht 17: 139-147.

Schmitz H. 1938 Conicera sensilipes n. sp. Male (Phoridae, Diptera). Mitteilungen über Hohlenund Karstforschung. Berlin 1938 (4): 43-48.

Schmitz H. 1953 Ungarische und andere paläarktische Phoriden des Ungarischen Nationalmuseums. Annales historico-naturales Musei nationalis hungarici (N.S.) 3: 203-211.

Schmitz H. 1955 33. Phoridae. In: Lindner E. (ed.) Die Fliegen der palaearktischen Region 4 (7). Lief. 180: 321-386.

Schmitz H. 1957 33. Phoridae. In: Lindner E. (ed.) Die Fliegen der palaearktischen Region 4 (7). Lief. 196: 417-464.

Schmitz H., Beyer E.M. 1965 33. Phoridae. In: Lindner E. (ed.) Die Fliegen der palaearktischen Region 4 (7). Lief. 258: 513-560.

Strobl G. 1898 [Fauna Diptera von Bosne, Herzegovine i Dalmacie]. Glasnik Zemaljskog Muzeja u Bosni i Hercegovini. Sarajevo 10: 387-466. (In Serbian and Latin)

Strobl G. 1900 Dipterenfauna von Bosnien, Hercegovina und Dalmatien. Wissenschaftliche Mitteilungen aus Bosnien und der Hercegovina 7: 552-670.

Strobl G. 1902 [New contributions to fauna of Diptera of the Balkan Peninsula]. Glasnik Zemaljskog Muzeja u Bosni i Hercegovini. Sarajevo 14: 461-517. (In Serbian and Latin) 